\title{
STRUCTURE AND DYNAMICS OF ELLIPTICAL GALAXIES
}

Tim de Zeeuw

Sterrewacht Leiden, Leiden, The Netherlands

\section{Marijn Franx ${ }^{1}$}

Center for Astrophysics, Cambridge, Massachusetts 02138

KEY WORDS: stellar kinematics, galaxy models, dark matter, galaxy formation

\section{INTRODUCTION}

Many questions remain outstanding regarding the structure, dynamics, and formation of elliptical galaxies. What are their intrinsic shapes? Do they have massive halos? Do they contain supermassive central black holes? What is the origin of the $r^{1 / 4}$ law? What is the origin of the "fundamental plane"? Are ellipticals dynamically similar to the bulges, or the halos, of spiral galaxies? What causes the distinction between ellipticals and spirals? When were elliptical galaxies formed, and on what time-scale? Was the main formation mechanism dissipationless, or did it involve gaseous processes? Was the substructure that is observed imprinted during formation, or is it more recent?

Historically, elliptical galaxies were thought to be dynamically simple, one-component collisionless stellar systems with a simple shape, containing very little gas (e.g. 130, 146). The big breakthrough came in the mid-1970s, when it became clear that rather than being boring dynamical systems, elliptical galaxies exhibit a rich variety of shapes and internal dynamics. They are not oblate isotropic rotators, but slowly tumbling triaxial stellar systems whose internal structure is not determined by just their total mass $M$, and total angular momentum $L(32,34-36,169)$. Thus, the observable

\footnotetext{
${ }^{1}$ Junior Fellow, Harvard Society of Fellows.
} 
properties of nearby elliptical galaxies contain information about their formation and, in principle, hold the key to answering many of the above questions. The current state of knowledge in this area is the topic of this review.

The structure of elliptical galaxies was reviewed previously by Gott (146) and Binney (38). More recent work can be found in the proceedings of IAU Symposium 127, which was devoted entirely to elliptical galaxies (83). The properties of ellipticals form too wide a topic to be reviewed in one paper. We refer readers interested in surface photometry, X-ray observations, and mergers to other reviews in this series $(193,111$, and 20 , respectively). Here we focus on the equilibrium dynamics of elliptical galaxies. In Section 2 we summarize recent work on dynamical models, with emphasis on triaxial systems. Observational efforts are reviewed in Section 3. Implications for the formation processes are discussed in Section 4. Finally, in Section 5 we outline possible future work.

\section{EQUILIBRIUM MODELS}

The dynamical time scale in an elliptical galaxy ranges from less than $10^{5}$ years in the central regions to more than $10^{9}$ years in the outskirts. With the possible exception of the inner nucleus, the two-body relaxation time is everywhere much larger than a Hubble time. Thus, an elliptical galaxy is a collisionless stellar system. The time scale for differential precession between neighboring orbits is usually of the order of 5-10 dynamical times and becomes comparable to a Hubble time beyond a few optical radii. As a result, the inner regions of elliptical galaxies are likely to be in equilibrium, in agreement with their regular and smooth optical appearance, but the outer regions probably have not experienced sufficient phase-mixing to have reached equilibrium.

The structure and dynamics of a collisionless stellar system are determined completely by the phase-space distribution function $f(\mathbf{r}, \mathbf{v}, t)$, which gives the distribution of the stars in the system over position $\mathbf{r}$ and velocity $\mathbf{v}$ as a function of time $t$. The distribution function must be non-negative, and satisfy the collisionless Boltzmann equation. In an equilibrium model $\partial f / \partial t=0$. Integration of $f$ over all velocities gives the density distribution $\rho(\mathbf{r})$ of the system. By Jeans' theorem, $f$ depends on the phase-space coordinates $\mathbf{r}$ and $\mathbf{v}$ only through the isolating integrals of motion admitted by the gravitational potential of the system $(179,220$, but see 40$)$. Any collisionless dynamical model can be scaled in mass, radius, and central velocity dispersion, but only two of these three parameters can be chosen freely, because of the constraint provided by the virial theorem (48). 


\subsection{Spherical and Axisymmetric Models}

A spherical potential admits four isolating integrals of motion: the orbital energy $E$ and the three components of the angular momentum vector $\mathbf{L}=\left(L_{x}, L_{y}, L_{z}\right)$. One usually considers models without any preferred axis, so that $f$ can depend only on $|\mathbf{L}|$, i.e. $f=f\left(E, L^{2}\right)$. Such models have anisotropic velocity distributions. It is possible to find the unique $f=f(E)$ that generates a given spherical density $\rho(r)$ by means of Eddington's inversion formula (103). If $\rho(r)$ falls off with radius sufficiently rapidly, this $f(E)$ is nowhere negative, and represents the unique isotropic model. Anisotropic models can sometimes be found by similar inversion methods (70), but they are usually constructed by assumption of a special functional form for $f\left(E, L^{2}\right)$ (e.g. 139, 236, 247, 323) or by numerical techniques $(280$, $290,294)$. Although few elliptical galaxies are spherical $(43,303)$, there is ample room for further work on the properties of anisotropic models. In particular, much information is contained in the line profiles, i.e. the distribution of stars as a function of line-of-sight radial velocity at different projected distances from the center. These profiles can now be measured $(25,122)$, but so far, few theoretical profiles have been calculated (71).

Axisymmetric potentials admit two exact isolating integrals, the energy $E$ and one angular momentum component, $L_{z}$, say. Lynden-Bell (219) generalized Eddington's formula and showed that for a given axisymmetric density $\rho(R, z)$, there is a unique distribution function $f\left(E, L_{z}^{2}\right)$. In such two-integral models, the velocity dispersion $\left\langle v_{R}^{2}\right\rangle$ in the equatorial plane is always equal to the perpendicular dispersion $\left\langle v_{z}^{2}\right\rangle$. The method for the actual calculation of $f$ is not easy to apply in practice, and only a few models have been constructed in this manner $(74,161,162,201)$. Other approaches include assuming a functional form for $f$ and using either series expansions $(199,259)$, or a numerical technique $(202,375)$ to find the associated density. The latter has been used recently to model the velocity fields of bulges of spiral galaxies $(177,178,296)$. More details on axisymmetric models can be found in Refs. $(70,82,84)$.

Orbits with $L_{z} \neq 0$ have a definite sense of rotation around the symmetry axis, but both clockwise and counterclockwise motion may occur. It follows that nonzero mean streaming is possible around the symmetry axis of any axisymmetric model and that its magnitude is not fixed by the density, since in each orbit the fraction of direct versus retrograde stars may be chosen freely. In two-integral models, $\left\langle v_{\phi}\right\rangle$ is often chosen so that the azimuthal dispersion $\left\langle v_{\phi}^{2}\right\rangle-\left\langle v_{\phi}\right\rangle^{2}=\left\langle v_{R}^{2}\right\rangle=\left\langle v_{z}^{2}\right\rangle$ (e.g. 302). Models of this kind are referred to as isotropic rotators. Other choices for $\left\langle v_{\phi}\right\rangle$ are discussed by Dejonghe (70).

Most orbits in realistic axisymmetric potentials are tubes around the 
symmetry axis and possess an extra isolating integral of motion, $I_{3}$ (e.g. $64,226,288)$. The remaining phase space is generally made up of a host of minor orbit families and irregular orbits. The latter do not have a third integral, and Binney has argued that for this reason Jeans' theorem is not valid for such systems $(40,275)$, thus implying that no true equilibrium solutions may exist. In many cases of interest, the fraction of irregular orbits turns out to be small, and they are nearly indistinguishable from regular orbits on time scales of the order of a Hubble time. For practical purposes, one may therefore probably still use Jeans' theorem, and construct approximate equilibrium models. The potentials relevant for elliptical galaxies are nearly spherical, and $I_{3}$ is related closely to $L^{2}(172,298)$. This fact has been used to construct approximate three-integral models with density profiles appropriate for elliptical galaxies $(271,272)$. Models based on special potentials that support an exact third integral are discussed in Section 2.3.

Kinematic properties of dynamical models are often calculated by direct solution of the Jeans equations, which relate the velocity dispersions to the density and the potential of the model $(48,180)$. Various solutions for spherical systems have been used to model the kinematics of elliptical galaxies, e.g. in connection with the search for nuclear black holes $(45$, 329, Section 3.5.1). An explicit solution of the Jeans equations is available also for two-integral axisymmetric models (163), but anisotropic axisymmetric solutions cannot be derived unless certain assumptions are made $(7,9,116$, Section 3.3.2). The resulting dispersion profiles are not always guaranteed to correspond to a distribution function $f \geq 0$, however.

\subsection{Triaxial Models Without Figure Rotation}

2.2.1 EXISTENCE Triaxial potentials generally admit only one exact isolating integral, the orbital energy $E$. Although there are three planes of reflection symmetry, there are no symmetry axes, and no component of the angular momentum vector is conserved. In a pioneering study, Schwarzschild $(305,306)$ showed by numerical orbit calculations that in triaxial potentials relevant for elliptical galaxies, most stellar orbits possess two effective integrals, $I_{2}$ and $I_{3}$, in addition to the energy. The fraction of irregular orbits is small (145). As a result, most orbits belong to one of only a few major families: box orbits, short-axis tube orbits, and long-axis tube orbits $(80,198)$. Tube orbits around the intermediate axis are unstable (153). The long-axis tubes come in two varieties, bringing the total number of major orbit families to four. Schwarzschild showed for a specific triaxial mass model - first with a stationary figure, and subsequently with a tumbling figure - that the individual orbital densities can be combined in the associated gravitational potential so that they reproduce the original mass 
model. This is equivalent to finding a distribution function $f$ that is consistent with the mass model (348), and hence Schwarzschild's work demonstrated that self-consistent triaxial galaxy models exist, with and without figure rotation. Similar conclusions were reached on the basis of N-body simulations $(2,248,250,251,371$, Section 2.7).

2.2.2 NON-UNIQUENESS The motion of a star on a box orbit in a nonrotating triaxial potential is a combination of oscillations in the three principal directions, so the orbit-averaged angular momentum vanishes. Stars on tube orbits have a definite sense of rotation around either the long axis or the short axis of the model. Clockwise and counterclockwise motion may occur in the same tube orbit. Because the fraction of direct versus retrograde stars may be chosen freely, the total angular momentum vector of a stationary triaxial model may be misaligned from the principal axes of the system: It may point anywhere in the plane containing both the long and the short axis. In Section 3.4 we examine the evidence for such a misalignment in the kinematics of elliptical galaxies.

It is also possible that different combinations of orbits with truly distinct shapes produce the same triaxial density distribution. Thus, there is a large degree of non-uniqueness in the distribution functions consistent with a given three-dimensional mass model. The purpose of the recent work on triaxial models is to explore this freedom in model building and to construct large sets of models that can be compared to observations. The main questions are: What are the permitted intrinsic shapes, figure rotation rates, and streaming velocities, and what constraints on the structure of elliptical galaxies can be deduced from detailed observations? We are still far from answering these questions satisfactorily, but many of the necessary tools have been developed. Specifically, much can be learned from a study of special models for which sufficient simplification occurs so that whole families of them can be studied. Two useful classes of such models are known. These are the separable or Stäckel models, and the scale-free models. We discuss each of these in turn.

\subsection{Separable Models}

Certain triaxial potentials admit three exact isolating integrals of motion. These are the potentials for which the Hamilton-Jacobi equation separates in specific orthogonal curvilinear coordinates. Special cases were discussed as many as 150 years ago (see 78 and 106 for historical references). The separable potentials have been named after Stäckel, who was the first to classify them systematically $(254,317,318)$. Their appearance in astronomy came through Eddington's seminal work on stellar dynamics (61, $102,221)$. The crucial insight that they correspond to smooth inhomo- 
geneous mass models is due to Kuzmin $(197,198)$. Their orbital structure resembles closely that of triaxial elliptical galaxies, and they are convenient prototypical models for these systems.

2.3.1 ORBITAL STRUCTURE The Hamilton-Jacobi equation separates in ellipsoidal coordinates (173) for the general Stäckel potential. The corresponding mass models have a nonrotating triaxial shape, with an arbitrary short-axis density profile, and arbitrary central axial ratios $(81,90$, 197, 198). On projection, the ellipticity of the isophotes generally changes with radius, but they show no twisting (121). All relevant models have cores with nonsingular density profiles. The integrals $I_{2}$ and $I_{3}$ are related to the angular momentum integrals of the axisymmetric and spherical limits (89). The three-dimensional orbital motion is a combination of three one-dimensional motions, each of which is either a libration or an oscillation in one of the three ellipsoidal coordinates. The orbits can be divided into four families: boxes, short-axis tubes, and two families of long-axis tubes (80). These are precisely the four major orbit families found in Schwarzschild's nonrotating triaxial model. Illustrations of the four orbital shapes have been presented by Statler $(48,320)$. The orbital structure simplifies in limiting cases with more symmetry (Figure 1). Prolate

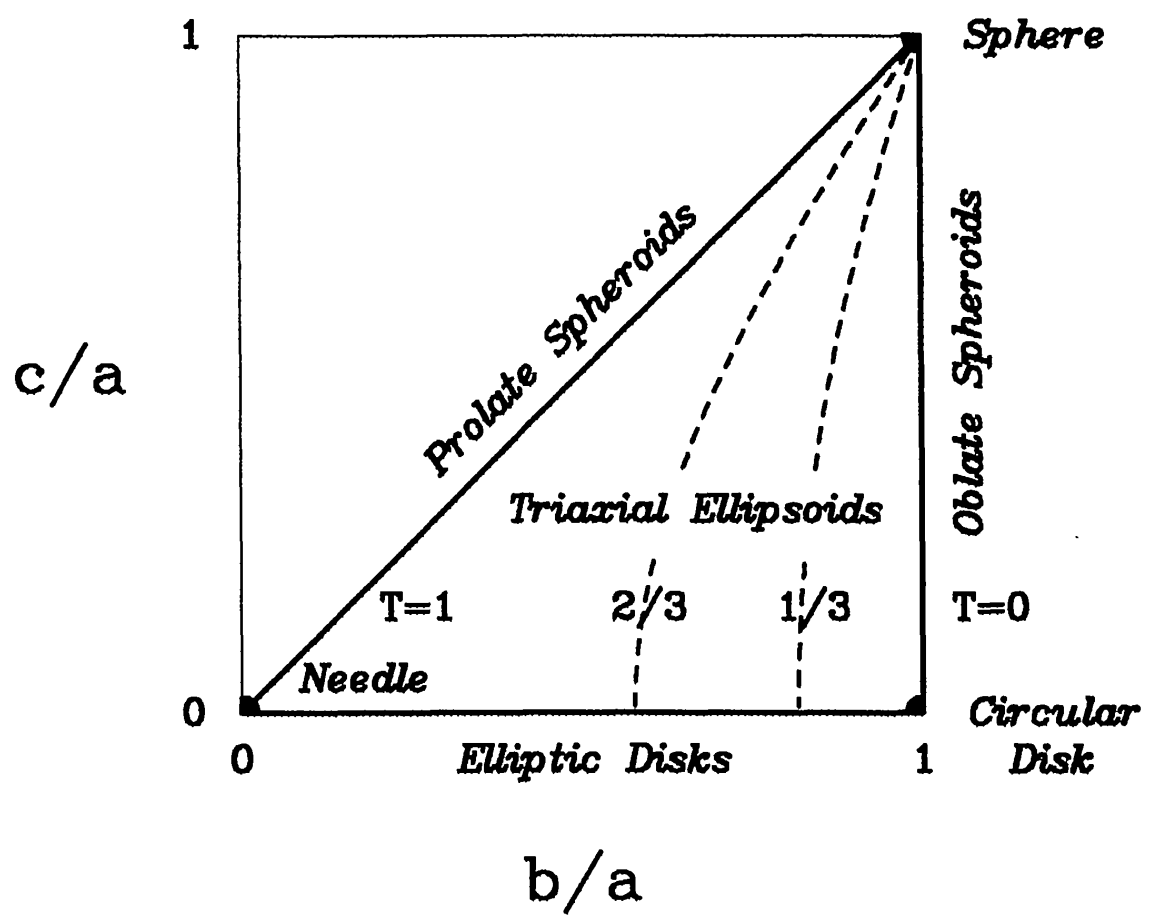

Figure 1 Ellipsoid Land: the plane of all possible axial ratios $b / a$ and $c / a$ for triaxial ellipsoids. The limiting cases with more symmetry are indicated. The dashed lines are curves of constant triaxiality $T$ (see Section 3.4). Oblate spheroids have $T=0$, prolate spheroids have $T=1$. 
Stäckel models support only inner- and outer-long-axis tubes, whereas all orbits are short-axis tubes in oblate separable models.

2.3.2 SELF-CONSISTENT MODELS The separable potentials support no irregular orbits, and therefore Jeans' theorem is strictly valid (40), so that $f=f\left(E, I_{2}, I_{3}\right)$. Direct calculation of distribution function(s) $f\left(E, I_{2}, I_{3}\right)$ consistent with a given triaxial density $\rho(x, y, z)$ by solving the fundamental integral equation - which gives $\rho$ as a triple integral of $f$ over the velocities - is a rather intimidating task $(49,74,75,110)$. The individual orbit densities in a separable model are known explicitly (80), however, so that building self-consistent models by means of Schwarzschild's method is straightforward. Statler (320) used this approach and constructed a large variety of different equilibrium models for a set of 21 triaxial separable models, all with the same density profile and with axial ratios covering all possible shapes. He found that the presence of four major orbit families, each of which can contribute density at any point, provides ample opportunity for exchanging orbits of different shapes while keeping the model density the same. As a result, the distribution functions for self-consistent separable triaxial models are highly nonunique (cf Section 2.2.2). This is reflected in the variety of kinematic properties displayed by Statler's models: The mean streaming motions range up to values that are comparable to those found for the fastest rotating ellipticals (Section 3.2), thus showing that ample mean streaming ("rotation") can occur in triaxial systems without figure rotation. Models with a large fraction of stars on box orbits show differences between the velocity dispersion profiles along the major and minor axes. Detailed kinematic observations may therefore help constrain the distribution functions of elliptical galaxies.

2.3.3 MAXIMUM STREAMING MODELS There is a special set of self-consistent separable models in which the full variety of box orbits is occupied, but only the thin tube orbits, i.e. those without any radial epicyclic motion, are populated. Their distribution functions can be found explicitly for separable models of arbitrary shapes and density profiles $(50,87,88$, $108,164-168,326)$. These models are hardly realistic, but they are useful because they allow the explicit calculation of the maximum possible stellar streaming velocities - and hence the maximum possible angular momenta around the long and the short axis - for any separable triaxial model. This is important for the interpretation of the observed velocity fields of elliptical galaxies, as it puts limits on the amount of streaming for a given triaxial shape.

2.3.4 KINEMATICS Eddington (102) already knew that the principal axes of the velocity ellipsoid in a Stäckel model are locally always aligned with 
the coordinate system in which the motion separates, so that in the central regions, the velocity ellipsoid is aligned along the principal axes of the model, whereas at large distances, it is aligned nearly radially. This pattern is seen also in nonseparable models with homogeneous cores (234). Solutions of the Jeans equations for axisymmetric separable models have been given recently $(74,107,109)$, but the equations for the triaxial case (218) have not been solved. They should provide useful information on the variety of dispersion profiles expected for equilibrium models, which is helpful in modeling kinematic data.

\subsection{Scale-Free Models}

The second set of special models that has received considerable attention in the last decade are those with density profiles that are single power-laws and have scale-free dynamical properties. This simplifies the construction of self-consistent equilibria, and is the main reason for the popularity of these models. Most studies have considered oblate and (nonrotating) triaxial models with $\rho \propto 1 / r^{2}$, which have a flat rotation curve and can be thought of as deformed singular isothermal spheres $(37,91,249,253,287$, 333).

Motion in the scale-free potentials is not separable. Numerical orbit calculations reveal that generally nearly all orbits are regular (288). The orbital structure of nonrotating triaxial models of this kind differs fundamentally from that of models with homogeneous cores, which include all the separable systems. The three families of tube orbits still exist, but the box orbits are replaced by a multitude of minor orbit families. These are associated with higher-order resonances between the oscillation frequencies along and perpendicular to the principal axes (140), and have been christened boxlets $(252,276)$. Although boxlets have a variety of shapes, it appears that in substantially flattened triaxial models, they cannot reproduce the characteristics of very elongated box orbits that remain close to the long axis. These are needed in any self-consistent model, because all tube orbits are elongated opposite to the figure of the model. The (preliminary) conclusion, based on inspection of orbits rather than on the actual construction of models, is that whereas exact oblate self-consistent scale-free models do exist $(213,214,287,289)$, flattened triaxial equilibria of this kind may not (210). The "scale-free" triaxial models that were built with Schwarzschild's method used orbits calculated in a potential that was strongly softened in the center, which reinstated most box orbits. As a result, these models are approximate, and not truly scale-free (215).

The appearance of boxlets is not confined to scale-free models. Other triaxial models that have been studied usually have density profiles that 
fall off proportionally to a power of $1 / r$ at large distances. Models that contain a central point mass have also received attention. Boxlets occur at large distances in many of these models, typically at 50-100 core radii $(136,140,210,276)$. On the basis of the various numerical experiments done to date, it appears that nonseparable potentials that support boxlets need to be avoided in building self-consistent models $(137,307)$. One way of doing this is to let the potential become at least axisymmetric at large radii, if not spherical, as in the case of the separable models (which do not have boxlets). Investigations of the orbital structure in various potentials that support boxlets should be supplemented with attempts to construct equilibria. This will provide valuable information on the possible shapes of the outer parts of elliptical galaxies and the halos of spirals.

\subsection{Figure Rotation}

Relatively little work has been done on the construction of triaxial galaxy models with figure rotation. A few special analytic models exist, but these do not have realistic density profiles $(127-129,160,161,345-347,350)$.

As is well known from the study of flat barred galaxy models (e.g. 65, $151,274,316,327$ ), figure rotation has significant consequences for the orbital structure (42). The available three-dimensional studies have concentrated mostly on tumbling around the short axis $(37,79,154,223,225$, $227,257,258,273)$. For slow tumbling rates, most orbits are regular; however, the directional nonuniqueness of stationary triaxial systems (Section 2.2.2) disappears in models with a rotating figure. The Coriolis force distinguishes between the clockwise and counterclockwise branch of an orbit, so that these branches have different shapes. Box orbits acquire a net angular momentum around the rotation axis (356), whereas long-axis tubes tip out of the plane containing the intermediate and short axes, with the two branches tipping in opposite directions, so that they have nonzero angular momentum about both the long and the short axes (306).

Schwarzschild was able to reproduce the density distribution of a slowly tumbling triaxial mass model with different combinations of orbits, thus establishing the nonuniqueness of such equilibrium models (306). This nonuniqueness is not surprising, as there now are no less than seven major orbit families (boxes, and three pairs of tubes). In order to obtain a symmetric figure, Schwarzschild had to populate the two branches of the two families of long-axis tubes in equal numbers, so that the total angular momentum pointed along the short axis. Conversely, this result implies that net streaming around the long axis gives measurable asymmetries (intrinsic twists) if the figure is rotating. This may provide a way to put an upper limit on the figure rotation of long-axis rotators (Section 3.4.1).

Rotating models have been produced also by N-body simulations (371). 
Van Albada (341) took the nonrotating triaxial endproduct of a simulation, applied a torque on the individual particles for a finite period of time, and found that he could generate stationary models with internal streaming, or tumbling models with internal streaming always in the direction of the tumbling; however, a larger variety of internal dynamics is probably possible. In the inner regions of the few self-consistent rotating models that have been constructed by Schwarzschild's method $(306,354)$, the mean streaming motions are retrograde in a corotating frame. Under favorable circumstances, an external, stationary observer may measure retrograde streaming, but only in the inner few core radii (355). Such counterrotation has been observed in a number of elliptical galaxies (Section 3.3.1).

\subsection{Stability}

An accurate delineation of the various instabilities that plague axisymmetric and triaxial equilibrium models, and the way in which these depend on the relative populations of the different orbit families, is of vital importance for our understanding of elliptical galaxies, because the requirement of stability will rule out many distribution functions. Not all viable galaxy models need to be dynamically stable, but if they are not, the growth rates of the instabilities must be of the order of a Hubble time. This important area of research is still largely unexplored. Our ignorance is caused in part by the lack of available models, and in part by the difficulty of the required calculations. Most results for systems that are not spheres or disks have been obtained by means of N-body simulations $(132,239,242,278)$.

Antonov showed that most isotropic spherical galaxy models are stable (5). He also realized that anisotropic spheres that are built mainly with radial orbits are dynamically unstable to the formation of a bar, i.e. become triaxial $(6,279)$. This fact was rediscovered a decade later by $\mathrm{N}$-body work (19), and stimulated a revival in this area of research $(30,76,144,228$, $243,265,266,268,299,349,361,362)$. The radial orbit instability is important for simulations of dissipationless collapse (Section 2.7), and also rules out certain models that have been fit to the photometric and kinematic observations of M87 (238, Section 3.5.1), thus illustrating that this type of instability might provide serious constraints on dynamical models for ellipticals. The radial orbit instability persists for nearly spherical triaxial models in which box orbits are heavily occupied (92), but disappears in elongated triaxial models with axial ratios of about 0.7 , in agreement with $\mathrm{N}$-body simulations. Schwarzschild's early triaxial models have axial ratios $1: 0.625: 0.5$, contain a large fraction of box orbits, and are stable (314).

Smooth distribution functions that can be used as initial conditions for 
accurate N-body simulations are available for the thin orbit models (Section 2.3.3). Merritt and his collaborators have undertaken a thorough study of their stability. Oblate thin orbit models with an axial ratio smaller than 0.33 are unstable to formation of rings $(93,246)$, in agreement with the well-known result that cold disks are similarly unstable $(267,331)$. All oblate thin orbit models are unstable to lopsided deformations (246). This instability decreases in strength for more nearly spherical models, and can be removed by increasing the radial pressure, i.e. by replacing the thin short-axis tubes by tubes with nonzero radial thickness. Very elongated prolate thin orbit models appear to be unstable against bending perturbations (245); again, the transition to stability occurs at an axial ratio of about 0.3 . No results are available yet for triaxial thin orbit models.

A number of instabilities appear to be responsible for limiting the shapes and internal velocity distributions of elliptical galaxies, in particular for the more flattened models (212). This is consistent with the hypothesis that the observed absence of elliptical galaxies flatter than E6 $(43,303)$ is due to dynamical instabilities (e.g. 132). Strongly flattened triaxial potentials generally support a substantial fraction of stochastic orbits, so their orbital structure is more complicated than that of the separable systems $(79,135$, 339). It is therefore not surprising that equilibrium models of this shape are unstable when perturbed. Hence, extending the stability studies to nonseparable models is important.

\subsection{Dissipationless Collapse}

Models for elliptical galaxies have been constructed also by means of $N$ body simulations, starting from initial conditions out of virial equilibrium (i.e. initial kinetic energy $T$ smaller than half the potential energy $W$ ). The resulting collapse generally produces a triaxial final state. Early work concentrated on establishing the existence of these systems $(2,248,250$, 251). Subsequent studies have addressed their internal dynamical structure (341, 371), including the growth of a central black hole (263), and the formation of shells $(20,281,282)$.

In a seminal paper, van Albada (340) showed that large collapse factors $(2 T / W \approx 0.1)$ and clumpy initial conditions are needed to produce (spherical) models with realistic $r^{1 / 4}$ density profiles (cf 232, 358), and that the properties of the final state depend on the dynamical constraints imposed during the collapse (229), as expected for incomplete violent relaxation $(222,324)$. Follow-up work has concentrated on better understanding the physics of this process, by using simple considerations to identify the probable form of the resulting distribution function $(39,174,247,323$, $335)$, or by invoking entropy arguments $(72,336,364,367)$. In simulations with larger collapse factors $(2 T / W<0.1)$, the radial orbit instability is 
important (243). The resulting final states are strongly prolate/triaxial (3, $4,216,217,269)$. They resemble elliptical galaxies in some respects, but their flattenings are somewhat larger than observed (Section 3.4). The presence of a dark halo does not change this result significantly (325).

\subsection{Conclusions}

The most important result of the work done in the past decade on the construction of equilibrium models is that many triaxial equilibria exist with a large variety of shapes and internal velocity structures. In particular, for a given shape there is freedom in the value of the (central) velocity dispersion, freedom in the direction of the total angular momentum, and freedom in the rate of figure rotation. Much work remains to be done on the construction of triaxial models, however-especially for those with tumbling figures - and on the study of their stability characteristics and observable properties.

\section{OBSERVATIONS}

Progress in the observational regime has been dramatic. CCDs have dramatically improved the reach of optical telescopes, and HST allows highresolution photometry of galactic nuclei. The Einstein satellite has opened up the way to X-ray observations of elliptical galaxies (111). Radio maps of $\mathrm{HI}$ are becoming readily available (351), and $\mathrm{CO}$ detections are increasing steadily (209).

The relevant observations for the delineation of the internal dynamical structure of elliptical galaxies are (maps of) the surface brightness distribution, radial velocity, velocity dispersion, absorption line profiles, and in some cases the velocity field of cold gas. X-ray observations provide quite independent information.

\subsection{Global Relations}

All self-consistent models can be scaled in radius and central velocity dispersion (Section 2). As the mass-to-light ratios of elliptical galaxies are not known a priori, correlations between global parameters of these galaxies provide constraints on their formation.

The first correlation that was found is the Faber-Jackson relation, between total luminosity $L$ and central velocity dispersion $\sigma_{\text {cen }}: L \propto \sigma_{\text {cen }}^{\alpha}$, with $3<\alpha<5$ (e.g. 96, 100, 113, 195, 328). The scatter in the relation is $\approx 0^{m} \cdot 6-0^{m} .7$ for the general field, but may be lower in specific clusters (e.g. $96,97,328)$. The equivalent result for spiral galaxies is the Tully-Fisher relation $L \propto v_{\text {circ }}^{\beta}$, with $3<\beta<5$, with a spread of $\approx 0^{m} \cdot 3-0^{m} .5(1,54,277$, $338)$. Both relations may deviate from pure power laws $(255,328)$. The 
origin of these relations is not well understood. It was initially thought that they are caused entirely by the perturbation spectrum prior to galaxy formation (148), but this mechanism might produce too much scatter, so a self-regulated process may be at work (365).

The Faber-Jackson relation has been superseded by the "fundamental plane." Two groups found independently that the scatter in the $L$ vs. $\sigma_{\text {cen }}$ relation decreases if a second parameter is included: $L \propto \sigma_{\text {cen }}^{2.65} r_{\mathrm{e}}^{0.65}$, with $r_{\mathrm{e}}$ the observed half-light radius $(94,95,97)$. Thus, galaxies do not fill the three-dimensional space of $L, \sigma_{\text {cen }}$, and $r_{\mathrm{e}}$, but lie on a two-dimensional surface in it: the fundamental plane. The existence of the fundamental plane implies that $M / L$ varies systematically with luminosity and surface brightness: $M / L \propto L^{0.25} \Sigma^{-0.05}(97)$. Similar relations exist for core luminosity and surface brightness $(112,207)$. The small scatter in the relations $\left(\approx 0^{m} .5\right.$ in $\left.L\right)$ is a real surprise: The measured velocity dispersion is the central velocity dispersion, which is sensitive to the prevailing anisotropy in the velocity distribution. As there is a large freedom in the possible anisotropies (Section 2), the central velocity dispersion can vary considerably even when the density profile is fixed (240, 293, 320). Apparently, elliptical galaxies show a much smaller range in anisotropies than allowed by the models.

If the observed $M / L$ values are consistent with a normal stellar population, the systematic variation of $M / L$ may be due to the physics of star formation $(112,149)$. We cannot, however, exclude the possibility that (nonbaryonic) dark matter contributes significantly in the central parts of galaxies. If this is the case, the fundamental plane would have to result from a "conspiracy" between the luminous and dark material to produce such similar $M / L$ values for a large variety of systems [extending from low mass ellipticals (262) to brightest cluster members].

Surprisingly, there is no conclusive evidence that any of the relations for ellipticals depend on environmental parameters (54). The residuals of the fundamental plane do not correlate with any of the other observed parameters like ellipticity or $\mathrm{Mg}_{2}$ index (112).

\subsection{Rotation}

Kinematic observations of elliptical galaxies have provided several surprises. First, it was found that luminous ellipticals rotate slowly (32, 169). A modern observation of NGC 1600 gives a rotational velocity of $1.9 \pm 2.3 \mathrm{~km} \mathrm{~s}^{-1}$ along the major axis, resulting in a $v / \sigma<0.013(182)$. Although in one particular case projection effects might play a role, the luminous ellipticals as a class have a mean $v / \sigma \approx 0.2$. Binney showed that this is significantly lower than expected for oblate isotropic rotators (35). Hence the shapes of bright elliptical galaxies are supported by anisotropies 
in the velocity distribution $(34,36)$. A useful diagnostic indicator is $(v / \sigma)^{*}$, which is the measured $v / \sigma$ divided by $(v / \sigma)_{\text {iso }}$, the value expected for oblate, rotationally flattened galaxies. A good approximation is $(v / \sigma)_{\text {iso }}=$ $[\varepsilon /(1-\varepsilon)]^{1 / 2}$, where $\varepsilon$ is the ellipticity of the galaxy (189). The parameter $(v / \sigma)^{*}$ is almost independent of inclination for oblate models. Bright ellipticals have a mean $(v / \sigma)^{*} \approx 0.4$.

The second surprise was the rapid rotation of spiral bulges, with $v / \sigma>0.5(117,171,194,231,370)$. Most observations have been done for (nearly) edge-on bulges in early-type spirals. Contamination of bulge light by light from the disk is a major problem, and many studies have tried to avoid this problem by observing at several position angles and/or offset slit positions. The total number of well-observed bulges is still relatively small. Detailed modeling, taking into account the influence of the disk, has shown that bulges rotate about as fast as expected for oblate isotropic rotators $(117,177,178,185)$.

The third surprise was the observation that ellipticals with $-18>M_{\mathrm{B}}>$ $-20^{m} 5$ have $(v / \sigma)^{*} \approx 0.9$, showing that intrinsically faint ellipticals rotate about as rapidly as bulges, most of which have comparable luminosities (68). There is a relation between luminosity and $v / \sigma$, in the sense that more luminous ellipticals rotate slower. The scatter about the relation is large, however, and its origin is not well-understood. Wyse \& Jones noted that $v / \sigma$ correlates with surface brightness, in the sense that galaxies with low surface brightness have lower $v / \sigma$ (376). They speculated that the high surface brightness galaxies had dissipated more energy during their formation, and have thus a higher rotation. This is not a complete explanation, as the specific angular momentum $J / M$ is also lower for the bright galaxies (27).

Subsequently, it was found that galaxies with "disky" isophotes have systematically high $v / \sigma(23,25,58)$. Galaxies with "boxy" isophotes have a large spread in $v / \sigma$. A possible explanation is that the galaxies with disky isophotes contain relatively luminous disks, as indicated by a recent statistical analysis of the available data (295). The disks may dominate the observed kinematics, because the measurement techniques are more sensitive to the low velocity dispersion system in a multicomponent galaxy (e.g. 122, 231, 370). Other parameters, like X-ray and radio-emission, are also correlated with the isophotal shape $(28,193)$. These correlations suggest that boxy ellipticals and disky ellipticals are intrinsically different and may have had a different formation history. Detailed analysis of isophote shapes and kinematic properties may help estimate the light contribution of the disks (e.g. 26, 295, 312).

Very recently, several groups have found slow rotation in dwarf ellipticals $(27,60,155)$. Bender \& Nieto (27) observed five faint ellipticals 
with $M_{\mathrm{B}}>-18^{m}$. All of these ellipticals have $v / \sigma<0.3$, and four have $(v / \sigma)^{*}<0.5$. The lower surface brightness galaxies generally are rotating slower. This result has added to the confusion in explaining the rotation of ellipticals. The low-mass and low-surface brightness systems may have formed in a different way. Supernova driven winds may have been more important during their formation (e.g. 77). These winds may help to decrease the $v / \sigma$ of a galaxy. Other types of explanations have been considered also (27).

Recent results on the rotation of globular cluster systems and planetary nebulae in the outer regions of elliptical galaxies indicate that their kinematics differ from that of the luminous central regions. Data on globular cluster systems in the two Virgo ellipticals M87 and NGC 4472 suggest that their $v / \sigma \approx 0.3$ (256), comparable to the $v / \sigma$ of the globular cluster system in our galaxy and Andromeda and consistent with the flattening of the luminous part of the galaxies. The number of observed globulars in these two cases is still small, and hence these results are very preliminary. The planetary nebula system in NGC 5128 (Cen A) is reported to be rotating with a velocity of $100 \mathrm{~km} \mathrm{~s}^{-1}$ at $4 r_{\mathrm{e}}$, as compared to $40 \mathrm{~km} \mathrm{~s}^{-1}$ for the luminous, inner, part of the galaxy $(119,372)$. This may be interpreted as a gradient in the rotational velocity, but it is also possible that the formation process has caused a systematic difference between the rotational properties of the different components.

We have collected the available high quality data from Refs. $(23,27,67$, $68,125,182)$. Photometric parameters and distances were taken from (114) or, if not available, from the references mentioned. The data are shown in Figure 2. All of these plots show a correlation and large scatter. In Figure $2 e$, the specific angular momentum is plotted against absolute magnitude. The solid line is the relation expected for galaxies with $v / \sigma=1$. There is a significant spread below this line for luminous galaxies.

At present, no satisfactory explanation can be given for these results. The systematic differences between low-mass and high-mass galaxies cannot be explained in a scenario of purely dissipationless formation (68). Simulations of dissipationless hierarchical formation predict a large scatter for $v / \sigma$, but no large systematic trend with mass $(18,378)$. The lower specific angular momentum of bright galaxies cannot be explained completely by differences in the dissipated energy during their formation.

The planetary nebula and globular cluster systems provide very interesting independent results. That they appear to rotate faster than the luminous part of the galaxy with which they are associated argues strongly against formation in a monolithic dissipative collapse. In such a picture the central parts are predicted to rotate faster than the outer parts. Merger simulations have shown that the inner parts of galaxies can lose their 

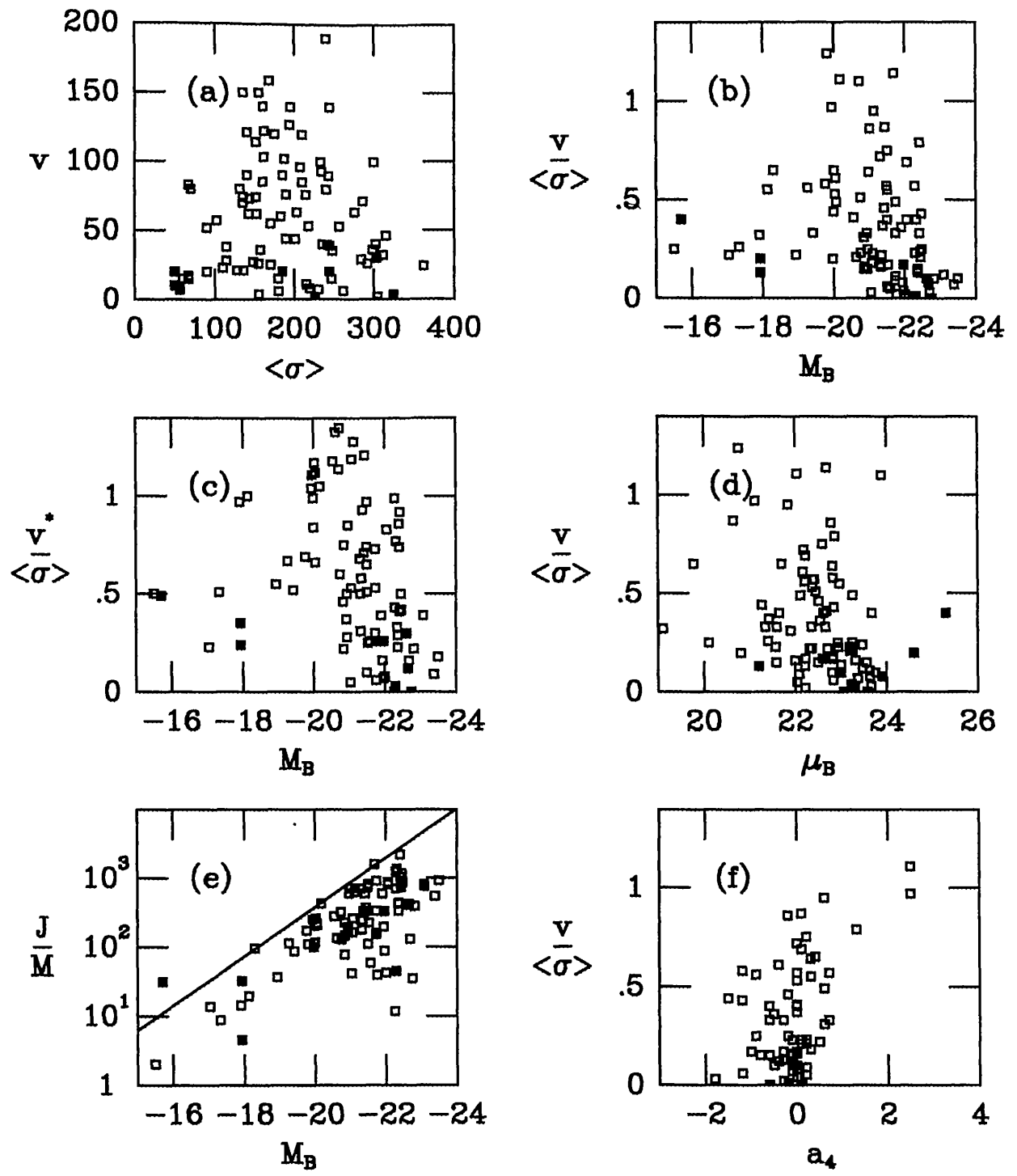

Figure 2 The rotational parameters of ellipticals correlated with dynamical and structural parameters. In $(a)$, the rotation velocity $v$ is plotted versus the mean velocity dispersion $\langle\sigma\rangle$, in $\mathrm{km} / \mathrm{s}$. Note the positive correlation for low $\langle\sigma\rangle$ and the negative correlation for high $\langle\sigma\rangle$, and the large scatter. The filled symbols are upper limits. In $(b)$ and $(c), v / \sigma$ and $(v / \sigma)^{*}$ are plotted against absolute magnitude. The most luminous galaxies rotate slowly, whereas intermediate galaxies rotate quickly, and the low-luminosity galaxies $\left(-M_{\mathrm{B}}<18\right)$ have intermediate rotation speed. In $(d), v / \sigma$ is plotted versus the surface brightness at an effective radius. Lower surface brightness galaxies rotate somewhat more slowly. In $(e)$, the specific angular momentum $J / M$ is plotted against absolute magnitude. $J / M$ is approximated by $c r_{\mathrm{e}} v$, with the constant $c$ arbitrarily set at 1 . The line is a fit to the distribution of points when $v$ is set equal to $\langle\sigma\rangle$. Note the large scatter below this line for the luminous galaxies (27). In $(f)$, $v / \sigma$ is plotted against the isophotal parameter $a_{4}$, which measures the deviations of the isophotes from ellipses (23). Disky isophotes give positive $a_{4}$, whereas boxy isophotes have negative $a_{4}$. 
angular momentum quickly to the outer parts $(15,17)$. The planetary nebula and/or globular cluster systems may have the same kinematics as the halo; this exciting possibility deserves great attention.

\subsection{Detailed Kinematics}

Improvements in detectors have allowed accurate measurements of the line-of-sight radial velocity and velocity dispersion with good spatial resolution. It was found that elliptical galaxies have complicated rotation curves, as exemplified by the discovery of kinematically distinct cores.

3.3.1 DISTINCT CORES The velocity fields of about $25 \%$ of all ellipticals show remarkable differences between the central regions and the outer parts $(25,170)$. Typical examples are NGC 5813 , which rotates rapidly in the center and shows almost no rotation in its outer parts (104); IC 1459 and NGC 5322, which rotate rapidly in the center and show opposite rotation along the major axis in the outer parts $(24,122)$; NGC 4365 and NGC 4406, which show rapid central rotation and slow rotation in the outer parts, but along the minor axis $(24,125,182,360)$. Whereas in these and other cases the core kinematics appears to be decoupled from that in the outer regions, the central rotation axis generally does not point in a random direction but is usually aligned with the minor axis. Only one example has been reported in which the rotation axis of the central parts lies along the major axis [NGC 5982 (360)]. Current observations can only detect velocity reversals in the inner parts of galaxies, as most data do not extend beyond one $r_{\mathrm{e}}$.

The velocity dispersion curves of the galaxies with distinct cores do not appear unusual, although in some cases $\sigma$ decreases toward the center. The photometry generally does not show marked peculiarities, and no strange color gradients have been reported (e.g. 126, 270).

The line profiles of the kinematically distinct cores show a strong asymmetry that reverses sign across the nucleus and is not due to stellar mismatch or instrumental effects [e.g. IC 1459 (122), NGC 5322 (25)]. Such line profiles arise naturally in a two-component galaxy, consisting of a slowly rotating main body with a large velocity dispersion and a small central component that counter-rotates rapidly and has a small velocity dispersion. This second component may therefore well be a disk. Such a cold dynamical component can influence strongly the observed rotation curve, even if its contribution to the observed light is as low as $20 \%$ (e.g. $122,231,370)$, but its effect on the velocity dispersion profiles is small.

Kormendy was the first to stress that the formation of these systems may be due to mergers (190). Specifically, he explained the observations of NGC 5813 by the hypothesis that a small galaxy had fallen into the 
center of a large galaxy, and that the central rotation reflected the rotation of the small galaxy. In this interpretation the light from the center comes mainly from the small galaxy. Thus the center is expected to be blue and to have a low velocity dispersion. Numerical simulations of this type of merger (12) have shown that the dynamical structure of the large galaxy is, in fact, changed significantly by the process of merging. The central rotation of the merger remnant corresponds to the orbital angular momentum, rather than the internal angular momentum, of the small infalling galaxy. The remnant of the small galaxy dominates the light in the central parts.

The disk-like structure of the subsystems suggests that they formed from gas that has settled to the center. This may have involved a starburst or a full-fledged merger of two spirals $(122,310)$. We can only put lower limits on the fractional mass needed to form these subsystems. Whether their formation is part of the formation of the whole galaxy or is a secondary event is impossible at present to decide. Observations of nearby mergers may help to elucidate the nature of the kinematic subsystems $(13,319)$.

Some elliptical galaxies show irregular rotation curves $(181,359)$, which may indicate that these systems are not yet fully relaxed. This result is unexpected, as the dynamical time in the central parts is quite short. Possibly, these systems can survive for much longer than a central dynamical time, or they form repeatedly, e.g. through regular infall of material, or by condensations and star formation from cooling flows.

\subsubsection{VELOCITY FIELDS Detailed kinematical "maps" have been obtained} for some ellipticals and bulges by combination of data taken at many slit positions $(44,171,175,194,231,373)$. Two-integral axisymmetric models can fit bulge velocity fields satisfactorily (178). The Jeans equations have been used to predict the apparent velocity dispersions and radial velocities under certain assumptions concerning the anisotropy of the velocity dispersions and the mass distribution (e.g. 44, 344). For some ellipticals, constant $M / L$ models with $f=f\left(E, L_{z}\right.$ ), i.e. $\sigma_{z}=\sigma_{R}$ (see Section 2.1), give satisfactory fits. The badly fitting galaxies may have distribution functions depending on three integrals, and/or changing $M / L$ as a function of radius (but see Section 3.5.2).

The purpose of these detailed studies is to obtain a better description of the distribution function. This might give useful information about the formation mechanism. The problems with this type of approach can be illustrated by considering the interpretation of the cylindrical rotation observed in box-shaped bulges and ellipticals $(175,194)$. Binney \& Petrou (47) modeled this with three-integral distribution functions that were sharply peaked, as expected from mergers or infall of cold systems (369). 
Rowley (296) constructed two-integral models, and concluded that the boxiness is caused by dissipative processes. Box-shaped bulges can be produced also by spin-up of spheroids (230). Yet another explanation is that boxy bulges are part of a bar (62). Observations of line profiles may help to distinguish between some of these suggestions. Detailed simulations will be extremely useful, even if they do not include gaseous processes.

\subsection{Intrinsic Shapes}

3.4.1 MINOR AXIS ROTATION Davies \& Birkinshaw found the first elliptical (NGC 4261) with strong minor axis rotation $\left(150 \mathrm{~km} \mathrm{~s}^{-1}\right)$ but almost no rotation along the major axis (66). Many more systems show significant rotation along the minor axis $(67,125,182,304,360)$. This rotation is impossible in an oblate axisymmetric system, and is a signature of triaxiality or prolateness $(41,63,188)$.

Minor axis rotation can be caused by two effects: First, for nearly all viewing angles, the projection of a triaxial galaxy has an apparent minor axis that is at a position angle that differs from that of the projected short axis of the galaxy. Hence, if the galaxy rotates intrinsically around its short axis, the observer will measure a gradient along the apparent minor axis. ${ }^{2}$ The geometric misalignment $\Theta_{\text {min }}$ between the apparent and intrinsic short axis is a function of the two axial ratios $b / a$ and $c / a$, and the two viewing angles $\phi, \theta$. Here $a, b$, and $c$ are the semi-axes, with $a \geq b \geq c$. The dependence of $\Theta_{\min }$ on the axial ratios can be simplified to $\Theta_{\min }(b / a$, $c / a, \phi, \theta)=\Theta_{\min }(T, \phi, \theta)$, where the triaxiality parameter $T$ is defined by $T=\left(1-b^{2} / a^{2}\right) /\left(1-c^{2} / a^{2}\right)(124)$. For oblate galaxies $T=0$, and for prolate galaxies $T=1$ (Figure 1).

Second, the total angular momentum of a triaxial galaxy can lie anywhere in the plane containing the long and the short axis (Section 2.2.2; 211). The observation that the minor axis rotation is small for many galaxies is surprising, since it shows that the angle $\psi_{\text {int }}$ between the intrinsic short axis and the angular momentum is generally small. Apparently, the formation mechanism produced a good (but not perfect?) alignment of the angular momentum and the intrinsic short axis. There is good indication that at least two galaxies rotate around their intrinsic long axis. NGC 4365 and NGC 4406 show minor axis rotation in their outer parts and major axis rotation in their inner parts. The $90^{\circ}$ misalignment between the inner and the outer parts suggests that one system rotates around the short axis and the other around the long axis. An analysis of the projection effects

\footnotetext{
${ }^{2}$ The rotation can be nonzero along the projected intrinsic short axis when the streamlines are noncircular.
} 
shows that the outer parts rotate around the long axis (125). Hence $\psi_{\text {int }} \neq 0$ for at least two galaxies.

Binney showed that a statistical analysis of minor axis rotation can constrain the intrinsic shapes of triaxial galaxies (41). Franx, Illingworth $\&$ de Zeeuw (124, FIZ) have extended his analysis and have applied it to the now available data. The three intrinsic parameters $b / a, c / a$, and $\psi_{\text {int }}$ are constrained by two observables: the apparent ellipticity $\varepsilon$ and the apparent misalignment $\psi$, defined by $\tan \psi=v_{\text {min }} / v_{\text {maj. }}$. A histogram of observed $\psi$ values is shown in Figure 3. The distribution of $\psi$ follows from that of $T$ and $\psi_{\text {int }}$, whereas the distribution of $\varepsilon$ is determined mainly by the distribution of $c / a$; it depends only weakly on $T$. FIZ inverted the $\varepsilon$ distribution to derive the distribution of $c / a$ for various assumptions about $T$. The resulting distribution shows a peak near $c / a=0.6-0.7$, and is zero or near-zero for round galaxies. The distribution of $\psi$ can be reproduced by a wide variety of models, even when a relation is assumed between $T$ and $\psi_{\text {int. }}$. A model of $\psi_{\text {int }}=0$ and a very smooth distribution of $T$ fits the data just as well as a model with $\psi_{\text {int }}=0$ and $56 \%$ of galaxies oblate and $44 \%$ of galaxies prolate. The mean triaxiality and mean intrinsic misalignment are better constrained: All solutions have $\langle T\rangle \leq 0.44$ and $\left\langle\psi_{\text {int }}\right\rangle \leq 25^{\circ}$. The larger $\left\langle\psi_{\text {int }}\right\rangle$, the smaller $\langle T\rangle$. These numbers are still uncertain, as the sample of galaxies is small and not complete in ellipticity.

3.4.2 OTHER TESTS The apparent surface brightness and velocity dispersion for a galaxy are expected to correlate with the apparent ellipticity, depending on the intrinsic shape of the galaxy. No conclusive results have been obtained from this type of test $(200,224,286)$. Merritt showed that high accuracy data are needed (235); recent results on the fundamental plane of galaxies $(94,97)$ indicate that galaxies are a true two-parameter

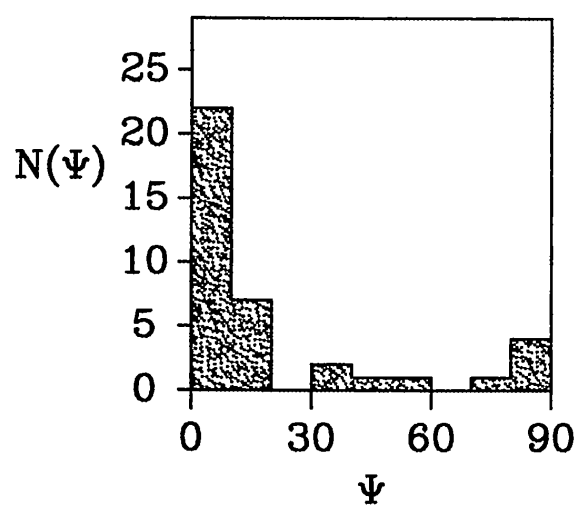

Figure 3 Histogram of apparent kinematic misalignment angle $\psi$. All galaxies with misalignments determined to $30^{\circ}$ or better are included (FIZ). 
family, thus making projection effects difficult to disentangle from intrinsic variations.

The interpretation of photometry alone is very uncertain. Isophotal twists are generally thought to be due to triaxial shapes $(373,374)$. The validity of this assumption is not certain, however, as some twists may be intrinsic (189). The correlation of twists with apparent ellipticity has been used to constrain the intrinsic shapes $(22,133)$, but these results are rather model dependent. The twists can constrain the change in triaxiality as a function of radius.

3.4.3 COLD GAS The kinematics of cold gas provides information on the intrinsic shape $(86,141,142,244,342)$. If the potential is not circular in the plane of a gas disk, the gas will be on elliptical orbits, and measurements of the surface brightness of the disk and its velocity field constrain the shape of the potential in the plane of the disk. If the gas has settled in a symmetry plane, the position angle offset between the stellar body and the gas disk provides an extra constraint on the shape. Data on NGC 5128 (372), NGC 1052 (69), NGC 4278 (208), NGC 5077 (31), and the bulge of NGC $5845(33,143)$ have been interpreted with gas on elliptic orbits in triaxial models. Most of the models assume that the gas has settled. The validity of this assumption is uncertain (85), as the settling time in the outer parts of galaxies can be rather long $(321,322)$, and Sparke has shown that self-gravitating rings or disks may never settle (315). Furthermore, the derived intrinsic shapes may not be representative, as the lifetime of the gas disk may depend on the shape of the galaxy.

\subsection{Mass-to-Light Ratios}

The determination of mass-to-light ratios from photometric and kinematic data alone is generally not unique $(45,292,329)$. Only if the run of the density profile with radius is assumed, as well as an intrinsic shape, is it possible to derive a global $M / L$ with the virial theorem $(8,29,38)$. It is impossible to derive the radial density profile from the velocity dispersion profile alone, given the freedom in the velocity anisotropy. It is nevertheless still possible to derive useful constraints on $M / L$ with this type of information $(184,237)$.

One might hope that the two-dimensional velocity and velocity dispersion fields constrain the $M / L$ value better. This needs to be verified with the help of three-integral axisymmetric and triaxial models, which show a great freedom in the velocity dispersion curves along the major and minor axes $(118,320)$.

Attempts to derive the radial $M / L$ profiles usually focus on one of two issues: (a) the presence of supermassive black holes in the nuclei of galaxies, 
and (b) the existence of dark matter in and around elliptical galaxies. We discuss both problems separately.

3.5.1 CENTRAL BLACK HOLES Most models of the energy production of QSOs invoke the presence of supermassive nuclear black holes. Predicted masses are in the range of $10^{8}$ to $10^{9} M_{\odot}$, depending on efficiencies and the average lifetime of the QSO phenomenon (284). If the lifetimes are short, many galaxies may have supermassive black holes.

High spatial resolution observations of nearby galaxies have indicated $M / L$ values that increase sharply in the nuclear regions. These are usually interpreted with supermassive black holes, but a cluster of dark remnants cannot be excluded for most cases (291). Nearby well-studied systems are M31 $(98,191,291)$ and M32 $(98,291,330)$. The inferred black hole masses are $10^{6}-10^{7} M_{\odot}$ and $10^{7}-10^{8} M_{\odot}$, respectively. A similar increase in $M / L$ was found for NGC $4594(176,192)$ and corresponds to a black hole mass of around $10^{9} M_{\odot}$. Surprisingly, the case is weakest for M87, a known active galaxy. The early claims of a detection of a central black hole (301, 377 ) were based mostly on surface photometry and velocity dispersions. It was soon realized that anisotropies in the velocity dispersion, and a nonstellar contribution to the light, can explain the observations with a constant $M / L$ model $(45,101,261)$. More recent data can be modeled with only a modest anisotropy in the velocity distribution $(73,99)$. The requirement that these models are stable provides an extra constraint (238), but the stability of the most recent models has not been studied.

The evidence for nuclear black holes in M31, M32, and NGC 4594 rests on curves of velocity dispersions and rotation. Modeling this type of data is not straightforward, as it involves the coupled deconvolution of the surface brightness, radial velocity, and velocity dispersion profiles. This makes searching the complete solution space difficult. The models employed are usually assumed to be spherical or axisymmetric (46), but to date no axisymmetric models based on explicit distribution functions $f \geq 0$ have been constructed. Additional information is contained in the line profiles $(71,233,290)$, which can now be derived from digital spectra. Application of this powerful technique may well put much tighter constraints on the behavior of $M / L$.

Gerhard has argued that the rapid central rotation in M31, M32, and NGC 4594 can be produced by a small bar that is viewed down its major axis (138). This hypothesis can be tested by observing a larger sample of galaxies, for which one would expect random viewing angles.

3.5.2 DARK MATTER Strong evidence for the presence of dark halos around spiral galaxies is provided by their H I rotation curves, but the 
evidence for dark matter around ellipticals is still rather poor. This topic was reviewed recently by Kent (186).

The velocity fields of emission-line gas disks have been used to determine $M / L$ profiles in the central regions of elliptical galaxies (e.g. 31, 55). Combination with stellar velocity dispersion profiles constrains the anisotropy in the velocity distribution, but the intrinsic shape of the galaxy has a significant effect on the modeling. This was demonstrated clearly for the E3 galaxy NGC 5077, where spherical models with the gas on circular orbits give a central decline in $M / L$, but triaxial models with constant $M / L$ are able to fit the data equally well (31). There is evidence, however, that the central gas rotation curves are suspect: The emission-line gas in many bulges often seems to rotate much slower than expected from the stellar velocity dispersions $(117,196,311)$. For NGC 4594, the gas velocities are smaller than the mean stellar streaming velocities (196). This may indicate that the gas is flowing in toward the center.

Few ellipticals contain substantial amounts of $\mathrm{H} \mathrm{I}$ out to large radii, e.g. NGC 1052 (352), NGC 2974 (187), NGC 4278 (208, 283), NGC 5128 (353), NGC 5666 (204), and IC 2006 (311). In NGC 1052, no radial change in $M / L$ is found, even though $\mathrm{HI}$ is observed as far out as $10 r_{\mathrm{e}}$. A strong outwards increase of $M / L$ was found for IC 2006, from $5 h_{50}$ in the inner parts to $16 h_{50}$ at $6.5 r_{\mathrm{e}}(311)$. Interpretation of these observations is complex, however, as ellipticals as a class are triaxial, so even if the gas has settled, it generally is not on circular orbits $(244,342)$. This was generally ignored in the past, but it influences the inferred $M / L$ behavior $(31,208)$.

The velocities of individual planetary nebulae and globular clusters can be measured to about $4 r_{\mathrm{e}}$, but they do not give strong constraints on $M / L$, because of the relatively small number of measurements $(119,158,159$, 256). Hot X-ray gas may well provide one of the best constraints on $M / L$ at large radii. Few temperature profiles are available, however, and as a result the mass determinations are still uncertain [e.g. 120, 300,337, and the review by Fabbiano (111)]. The new data from ROSAT may help to improve this situation.

A completely different constraint on the dark halos comes from cluster dynamics. Merritt has argued that no more than $15 \%$ of the mass in clusters can be attached to individual galaxies (241). This implies that the total $M / L$ for a galaxy is 0.15 times the $M / L$ of the total cluster (typically $200 h_{50}$ ), resulting in $M / L \leq 30 h_{50}$. This would imply that the halos contain less than three times the mass of the luminous parts. This rather low factor may result from stripping during the formation of the cluster; it may very well be that the progenitors of ellipticals had heavier halos. Furthermore, Merritt's result shows that in order to answer the question of whether 
elliptical galaxies have dark halos, one has to demonstrate that any dark matter found is in fact related to the galaxy and has a similar velocity dispersion.

\section{FORMATION PROCESSES}

The most general conclusion that can be drawn from a comparison of the dynamical models with the data is that elliptical galaxies show a much narrower range in structure and kinematics than allowed by the models. The clearest examples are the similar light profiles and $M / L$ values, the Faber-Jackson relation, the regular isophote shapes of galaxies, the correlations of $v / \sigma$ with luminosity and other parameters, the relatively small kinematic misalignments, the color-magnitude relation (193), etc. These properties are most likely a result of the formation process and not the equilibrium dynamics. It should be noted in this context that the role of instabilities has not been explored fully.

Our understanding of galaxy formation has not progressed sufficiently to have resulted in a finite number of competing theories and predictions, which can be compared in detail to the observations. Some elements of theories and observations are usually pieced together, often without the basis of a comprehensive model of galaxy formation. Given the lack of predictive power for physical processes involving gas dynamics and star formation, many of the statements we can make are only qualitative.

A fundamental problem with any interpretation is the question of whether ellipticals have dark halos, and if not, if that lack is due to stripping. If ellipticals were born without dark halos, then they are very different from spiral galaxies, and the similarity between bulges and ellipticals would only be superficial. Without any good evidence for such a fundamental distinction, it is probably best to assume that both types of galaxies have dark halos.

\subsection{Which Processes Dominated the Formation of Galaxies?}

The formation processes can be divided into two categories: dissipationless processes, such as the formation of structure in pure cold dark matter models, and dissipational processes, which include star formation, cooling and heating of gas, etc. In an entirely dissipationless scenario, most of the observables can be related directly to the initial conditions. In a dissipational scenario, the physics of star formation, galactic winds, etc, determine many of the observables.

The $r^{1 / 4}$-law profiles can be produced entirely by dissipationless collapse or mergers (Section 2.7). Simulations have shown that realistic profiles result from a wide variety of initial conditions $(4,232,340,357,363,364)$. 
Most of these simulations did not distinguish between dark matter and luminous matter, and are somewhat difficult to interpret directly. Simulations of mergers between disk galaxies with dark halos produce realistic ellipticals $(15-17,115,134,260)$.

The scenarios with dissipationless hierarchical formation do not produce the current luminosity function directly. The typical structures formed at the present epoch are not galaxies but groups and clusters of galaxies. As substructure tends to be erased in these scenarios, dissipational processes must have played a role (e.g. 368). The cooling characteristics of the baryonic matter are most often used to explain the typical galactic masses $(285,313,366,368)$. Recent simulations including dissipation were successful at preserving galaxies during the cluster collapse (57). Dissipational scenarios in which galaxies form from shocked gas usually invoke the same hierarchical process to explain the typical galactic mass $(21,105)$.

As discussed in Section 3.1, the origin of the Faber-Jackson relation and the fundamental plane is not entirely clear. One of the main uncertainties is the dark matter content of the luminous parts. These problems are related to the formation of spiral galaxies with their flat rotation curves (10). The formation scenarios generally predict a significant dark matter content for spirals in the visible regions $(14,51,297)$. By analogy, the same may hold for ellipticals.

The color gradients and the color-magnitude relation for ellipticals no doubt must be explained in terms of gaseous processes, but the exact mechanism is unknown. Radial inflows, galactic scale winds, and the hot $\mathrm{X}$-ray gas envelopes probably play a role $(56,123,152,205,206)$.

The slow rotation of bright ellipticals can be explained by a dissipationless merger scenario $(15,17)$. The rapid rotation of faint elliptical galaxies and the correlations of $v / \sigma$ with various parameters are more difficult to understand. There is a wide range of parameters in a merger (e.g. relative masses, bulge/disk ratios, gas fractions, orbital parameters), so that scatter in the properties of the merger remnants can be expected. Whether systematic changes of $v / \sigma$ can be produced remains to be seen.

The analysis of the intrinsic shapes of elliptical galaxies may provide equally interesting information. The predictions from dissipationless simulations are usually more triaxial than permitted by the data (124). Both the data sample and the analysis of the simulations should be improved before we can draw firm conclusions. If this trend is confirmed, however, we may need the inclusion of gas dynamics to explain the discrepancy. Gas disks may be able to deform the galaxies, as the gas tends to settle in an elliptical disk, which is elongated perpendicular to the main body of the galaxy. 
We conclude that both gaseous and collisionless processes played an important role during galaxy formation, and determined many of the observed properties. Significant progress has been made toward modeling of dissipationless processes. The challenge is to achieve a similar level of sophistication for gaseous processes, as these are crucial for our understanding of many of the fundamental parameters.

\subsection{Mergers?}

Many formation scenarios invoke a hierarchical build-up of collapsed clumps to typical galaxy masses $(21,105,368)$. There appears to be supporting evidence that (many) ellipticals have formed in a hierarchical way. The presence of shells and kinematically distinct cores, and the low rotation of bright ellipticals are expected in a hierarchical formation scheme. Detailed studies of nearby merger remnants have shown that these systems are remarkably similar to elliptical galaxies $(203,308,309)$. Furthermore, as pointed out by Toomre, the present merger rate of spiral galaxies is high enough to have produced a significant fraction of current ellipticals during the lifetime of the universe (332).

Most of the arguments against merging apply to very simple scenarios in which, for example, ellipticals were formed from low-luminosity ellipticals, or from gas-poor spirals (264). Considerable evidence suggests that the gas content of galaxies was different at early epochs, so arguments against forming ellipticals from current spirals (including gas) may say more about the epoch at which galaxies formed than about the mechanism by which they formed $(150,206,343)$. The globular cluster frequencies of galaxies may provide interesting constraints $(150,343)$, but, unfortunately, we have a very poor understanding of the formation and evolution of these systems (cf. the completely different formation scenarios discussed in 131 and 183).

Merger simulations are extremely useful because of their predictive power. For this reason, they are an excellent paradigm against which current observations can be tested. The simplest way to include gaseous processes in the simulations is by assuming that the gas formed a disk before the merger event and is (partially) expelled afterwards. This type of scenario is now being investigated by Barnes (15-17). Detailed comparison of his predictions with observed quantities will be very interesting. The main uncertainties of this scenario concern the properties of the galaxies before the merger (especially their gas content), however, and the question of whether galaxies assemble their mass in one big event or in many, more gentle, events. Simulations of galaxy formation (e.g. 57) and observations of high redshift galaxies may provide useful additional information. 


\subsection{When Did Ellipticals Form?}

The dynamics of equilibrium models do not give constraints on when ellipticals formed. Kinematically distinct cores may persist for at least a relaxation time, which is larger than a Hubble time. The original arguments for a very fast collapse and formation of ellipticals came from estimates of the free-fall time of galaxies with the assumption of a small collapse factor (146). Since the discovery of dark matter (and the resulting decrease in mean density and increase in free-fall time) and the realization that galaxy formation may proceed in a hierarchical way, the estimates of the formation epoch have varied between redshifts of 30 to redshifts of 1 or less $(21,365)$. Furthermore, the formation process may be extended in time, even for spheroids.

The nonequilibrium structures in elliptical galaxies, such as shells, tidal tails, and asymmetric rotation curves, give independent information on the formation epoch, or on merger rates and mass accretion rates (11, 332). If measured accurately, these can constrain simulations of galaxy formation (57). Important information may come from studies that combine deep photometry with an analysis of the stellar populations $(59,310)$. This will shed light on star formation during merger events, and may help in detecting older merger remnants.

\subsection{Galaxy Formation at $\mathrm{z}=0$ ?}

Given the difficulty in observing high redshift galaxies, we may ask which aspects of the formation process we can see in the nearby universe. Ongoing mergers of spiral galaxies provide unique laboratories for a study of the physics of encounters, and they have been modeled in detail by various authors $(52,53,157,334)$. The current simulations are very successful in reproducing most observables, and can be used to constrain the orbits and halo properties of the merging galaxies. The end results of these encounters are expected to resemble bright elliptical galaxies $(15,17)$.

One of the most interesting aspects of investigations of this type is that they may result in a better understanding of the gas dynamics and star formation during the encounter. Hernquist has shown that gas can be driven into the centers of galaxies during the encounter (156). This provides a natural mechanism for nuclear starbursts and/or nuclear activity. The gas masses and star formation rates in starbursts can be quite high, and these events may be typical of the events that formed the stars in most galaxies.

\section{FUTURE WORK}

The formation of galaxies is an integral part of the problem of formation of structure in the Universe. As such, we should not expect to find a 
solution to the problem of galaxy formation by looking at just galaxies. Nevertheless, galaxies as building blocks and tracers are so important that we have to understand their properties well before we can fully model the larger scale structures. The main conclusion from recent work on elliptical galaxies is that they are complex systems. Their population may have undergone significant evolution over an extended period in the lifetime of the Universe. We are still far from a complete understanding of their structure and formation. There is an immediate need for good theoretical simulations of formation processes that will help with interpreting the observations. Below we list specific areas of research in which significant progress can be made.

\subsection{Equilibrium Models and Stability}

A large set of self-consistent triaxial models is needed to help improve the interpretation of the detailed photometric and kinematic observations of elliptical galaxies. Separable as well as scale-free models should be part of this set. Whether or not the intrinsic shapes of the latter models are constrained by the orbital structure is important to establish. Furthermore, the stability of these models should be explored in detail. This is best done with a hybrid strategy in which $\mathrm{N}$-body simulations are complemented with semi-analytic approaches such as the calculation of normal modes via the matrix method (362).

\subsection{Observations}

Better limits on the presence of dark halos around elliptical galaxies are crucial. We need to know the typical masses and velocity dispersions of these halos to distinguish between galaxy-size halos and dark matter in clusters. Some of the tracers (globular clusters, planetary nebulae) that have been used for this purpose also give very interesting information on the rotation of the outer regions, which may provide unique tests for some formation scenarios. The detection of black holes in the nuclei of ellipticals remains a pressing issue that awaits high-resolution imaging and spectroscopy. The presence of substructure in elliptical galaxies should be further investigated. Typical masses and dynamical alignments/antialignments can provide useful constraints on their formation. The division between elliptical galaxies and $\mathrm{S} 0$ galaxies has become fuzzier, as many ellipticals are now known to have weak disks (193). Detailed studies of S0s will provide interesting information on the similarity of these types of systems. The dependence of the global properties on environment is an area of research that has not been explored yet. 


\subsection{Formation Processes}

Collisionless merger simulations provide important predictions for the properties of elliptical galaxies, and they need to be exploited as fully as possible. A detailed analysis of the internal structure of the resulting galaxy models as a function of initial conditions is crucial for a good interpretation of the data. Our basic hypothesis that galaxies are not evolving on a time scale smaller than the Hubble time can also be tested with these techniques.

Dissipational processes clearly do play a role in the formation of ellipticals, and the eventual inclusion of gas dynamics, star formation, and galactic winds, etc is needed. Full modeling of this kind appears to lie far in the future, but the first attempts in this direction have shown their power already (156). This approach should be directed also toward the simulation of nearby observable phenomena, such as starburst galaxies and galactic scale winds (152). Comparisons of observations of these energetic events with detailed simulations will clarify their nature, and will also provide good tests of the numerical codes.

\subsection{Evolution of Galaxies}

Big telescopes and space observatories will allow us to study intermediate redshift and high redshift galaxies in more detail in the future. The studies of their dynamics, structure, and stellar populations will provide direct insight into the evolution of galaxies.

\section{ACKNOWLEDGMENTS}

It is a pleasure to thank Garth Illingworth and Martin Schwarzschild for fruitful collaborations and many stimulating discussions. We thank Ralf Bender, Giuseppe Galletta, Steve Kent, John Kormendy, Joanna Lees, Reynier Peletier, Paul Schechter, Alar Toomre, Roeland van der Marel, Jacqueline van Gorkom, and many other colleagues for lively discussions and for making data available in digital form. M. F. acknowledges support from the Harvard Society of Fellows, the Harvard College Observatory, and the Leids Kerkhoven-Bosscha Fonds. The hospitality of the Aspen Center for Physics, where the initial part of this work was done, is gratefully recorded.

\section{Literature Cited}

1. Aaronson, M., Mould, J. R. 1983. Ap. J. 265: 1-17

2. Aarseth, S., Binney, J. J. 1976. MNRAS 185: 227-43

3. Aguilar, L. A. 1988. Celest. Mech. 41: 3-37

4. Aguilar, L. A., Merritt, D. R. 1990. Ap. J. 354: 33-51

5. Antonov, V. A. 1962. Vestnik Leningrad Univ. 19: 96-111 (Transl. in Ref. 83, pp. 531-48)

6. Antonov, V. A. 1973. In Dynamics of Galaxies and Clusters, ed. T. B. Omarov, pp. 139-43. Alma Ata: Akad. Nauk Kaz. SSR (Transl. in Ref. 83, pp. 549-52) 
7. Bacon, R: 1985. Astron. Astrophys. 143: 84-93

8. Bacon, R., Monnet, G., Simien, F. 1985. Astron. Astrophys. 152: 315-24

9. Bacon, R., Simien, F., Monnet, G. 1983. Astron. Astrophys. 128: 405-10

10. Bahcall, J., Casertano, S. 1985. Ap. J. 293: L7-10

11. Bahcall, S., Tremaine, S. D. 1988. Ap. J. Lett. 326: L1-4

12. Balcells, M., Quinn, P. J. 1990. Ap. J. 361: 381-93

13. Balcells, M., Stanford, S. A. 1990. Ap. J. 362: 443-48

14. Barnes, J. E. 1987. In Nearly Normal Galaxies, ed. S. M. Faber, pp. 154-60. Berlin: Springer-Verlag

15. Barnes, J. E. 1988. Ap. J. 331: 699 717

16. Barnes, J. E. 1989. Nature 338: 123-26

17. Barnes, J. E. 1990. In Dynamics and Interactions of Galaxies, ed. R. Wielen, pp. 186-95. Berlin: Springer-Verlag

18. Barnes, J. E., Efstathiou, G. 1987. Ap. J. 319: 575-600

19. Barnes, J. E., Goodman, J., Hut, P. 1986. Ap. J. 300: 122-31

20. Barnes, J. E., Hernquist, L. 1992. Annu. Rev. Astron. Astrophys. 30: In press

21. Baron, E., White, S. D. M. 1987. Ap. J. 322: 585-96

22. Benacchio, L., Galletta, G. 1980. MNRAS 193: 885-94

23. Bender, R. 1988. Astron. Astrophys. Lett. 193: L7-10

24. Bender, R. 1988. Astron. Astrophys. Lett. 202: L5-8

25. Bender, R. 1990. In Dynamics and Interactions of Galaxies, ed. R. Wielen, pp. 232-43. Berlin: Springer-Verlag

26. Bender, R. 1990. Astron. Astrophys. 229: 441-51

27. Bender, R., Nieto, J. L. 1990. Astron. Astrophys. 239: 97-112

28. Bender, R., Surma, P., Döbereiner, S. Möllenhof, C., Madejsky, R. 1989. Astron. Astrophys. 217: 35-43

29. Bertin, G., Saglia, R. P., Stiavelli, M. 1988. Ap. J. 330: 78-90

30. Bertin, G., Stiavelli, M. 1989. Ap. J. 338: 723-34

31. Bertola, F., Bettoni, D., Danziger, I. J., Sadler, E. M., Sparke, L. S., de Zeeuw, P. T. 1991. Ap. J. In press

32. Bertola, F., Capaccioli, M. 1975. Ap.J. 200: 439-45

33. Bertola, F., Rubin, V. C., Zeilinger, W. W. 1989. Ap. J. Lett. 345: L29-32

34. Binney, J. J. 1976. MNRAS 177: 19-29

35. Binney, J. J. 1978. MNRAS 183: 50114

36. Binney, J. J. 1978. Comments Astrophys. 8: 27-36
37. Binney, J. J. 1981. MNRAS 196: 45567

38. Binney, J. J. 1982. Annu. Rev. Astron. Astrophys. 20: 399-429

39. Binney, J. J. 1982. MNRAS 200: 95164

40. Binney, J. J. 1982. MNRAS 201: 15-19

41. Binney, J. J. 1985. MNRAS 212: $767-$ 81

42. Binney, J. J. 1987. In Ref. 83, pp. 229 39

43. Binney, J. J., de Vaucouleurs, G. 1981. MNRAS 194: 679-91

44. Binney, J. J., Davies, R. L., Illingworth, G. D. 1990. Ap. J. 361: 78-97

45. Binney, J. J., Mamon, G. A. 1982. MNRAS 200: 361-75

46. Binney, J. J., Petit, J.-M. 1989. In Dynamics of Dense Stellar Systems, ed. D. Merritt, pp. 43-55. Cambridge: Cambridge Univ. Press

47. Binney, J. J., Petrou, M. 1985. MNRAS 214: 449-62

48. Binney, J. J., Tremaine, S. D. 1987. Galactic Dynamics. Princeton, NJ: Princeton Univ. Press

49. Bishop, J. L. 1986. Ap. J. 305: 14-27

50. Bishop, J. L. 1987. Ap. J. 322: 618-31

51. Blumenthal, G. R., Faber, S. M. Flores, R., Primack, J. R. 1986. Ap. J. 301: 27-34

52. Borne, K. D. 1988. Ap. J. 330: 61-77

53. Borne, K. D. 1990. In Dynamics and Interactions of Galaxies, ed. R. Wielen, pp. 196-99. Berlin: Springer-Verlag

54. Burstein, D., Faber, S. M., Dressler, A. 1990. Ap. J. 354: 18-32

55. Caldwell, N., Kirshner, R. P., Richstone, D. O. 1986. Ap. J. 305: 136-47

56. Carlberg, R. G. 1984. Ap. J. 286: 40315

57. Carlberg, R. G., Couchman, H. M. P. 1989. Ap. J. 340: 47-68

58. Carter, D. 1987. Ap. J. 312: 514-17

59. Carter, D., Prieur, J. L., Wilkinson, A., Sparks, W. B., Malin, D. F. 1988. MNRAS 235: 813-25

60. Carter, D., Sadler, E. M. 1990. MNRAS 245: 12P-16

61. Chandrasekhar, S. 1942. Principles of Stellar Dynamics. Chicago: Univ. Chicago Press

62. Combes, F., Debbash, F., Friedli, D., Pfenniger, D. 1990. Astron. Astrophys. 233: 82-95

63. Contopoulos, G. 1956. Z. Astrophys. 39: $126-32$

64. Contopoulos, G. 1960. Z. Astrophys. 49: 273-91

65. Contopoulos, G., Papayannopoulos, Th. 1980. Astron. Astrophys. 92: 33-46

66. Davies, R. L., Birkinshaw, M. 1986. Ap. J. Lett. 303: L45-49 
67. Davies, R. L., Birkinshaw, M. 1988. Ap. J. Suppl. 68: 409-47

68. Davies, R. L., Efstathiou, G., Fall, S. M., Illingworth, G. D., Schechter, P. L. 1983. Ap. J. 266: 41-57

69. Davies, R. L., Illingworth, G. D. 1986. Ap. J. 302: 234-44

70. Dejonghe, H. 1986. Phys. Rep. 133: 217-313

71. Dejonghe, H. 1987. MNRAS 224: 13 39

72. Dejonghe, H. 1987. Ap. J. 320: 47781

73. Dejonghe, H. 1989. In Dynamics of Dense Stellar Systems, ed. D. Merritt, pp. 69-74. Cambridge: Cambridge Univ. Press

74. Dejonghe, H., de Zeeuw, P. T. 1988. Ap. J. 333: 90-129

75. Dejonghe, H., Laurent, D. 1991. $M N R A S$. In press

76. Dejonghe, H., Merritt, D. R. 1988. Ap. J. 328: 93-102

77. Dekel, A., Silk, J. 1986. Ap. J. 303: 3955

78. de Zeeuw, P. T. 1984. Dynamics of triaxial stellar systems. $\mathrm{PhD}$ thesis. Leiden Univ.

79. de Zeeuw, P. T. 1985. MNRAS 215: 731-60

80. de Zeeuw, P. T. 1985. MNRAS 216: 273-334

81. de Zeeuw, P. T. 1985. MNRAS 216: 599-612

82. de Zeeuw, P. T. 1987. In IAU Symp. 127, Structure and Dynamics of Elliptical Galaxies, ed. P. T. de Zeeuw, pp. 271-90. Dordrecht: Reidel

83. de Zeeuw, P. T., ed. 1987. IAU Symp. 127: Structure and Dynamics of Elliptical Galaxies. Dordrecht: Reidel

84. de Zeeuw, P. T. 1988. In Integrability in Dynamical Systems, Ann. NY Acad. Sci. 536: 15-24

85. de Zeeuw, P. T. 1990. In Dynamics and Interactions of Galaxies, ed. R. Wielen, pp. 263-66. New York: Springer-Verlag

86. de Zeeuw, P. T., Franx, M. 1989. Ap. J. 343: $617-43$

87. de Zeeuw, P. T., Hunter, C. 1990. Ap. J. 356: 365-88

88. de Zeeuw, P. T., Hunter, C., Schwarzschild, M. 1987. Ap. J. 317: 607-36

89. de Zeeuw, P. T., Lynden-Bell, D. 1985. MNRAS 215: 713-30

90. de Zeeuw, P. T., Peletier, R., Franx, M. 1986. MNRAS 221: 1001-22

91. de Zeeuw, P. T., Pfenniger, D. 1988. MNRAS 235: 949-96

92. de Zeeuw, P. T., Schwarzschild, M. 1989. Ap. J. 345: 84-100

93. de Zeeuw, P. T., Schwarzschild, M. 1991. Ap. J. 369: 57-78
94. Djorgovski, S., Davis, M. 1987. Ap. J. 313: 59-68

95. Djorgovski, S., de Carvalho, R., Han, M. S. 1988. In ASP conference series, Vol. 4, The Extragalactic Distance Scale, ed. S. van den Bergh, C. J. Pritchet, pp. 329-41. Provo: Brigham Young Univ. Print Serv.

96. Dressler, A. 1984. Ap. J. 281: 512-24

97. Dressler, A., Lynden-Bell, D., Burstein, D., Davies, R. L., Faber, S. M. Terlevich, R. J., Wegner, G. 1987. Ap. J. 313: 42-58

98. Dressler, A., Richstone, D. O. 1988. Ap. J. 324: 701-13

99. Dressler, A., Richstone, D. O. 1990. Ap. J. 348: 120-26

100. Dressler, A., Sandage, A. 1983. Ap. J. 265: 664-80

101. Duncan, M. J., Wheeler, J. C. 1980. Ap. J. Lett. 237: L27-31

102. Eddington, A. S. 1915. MNRAS 76: 37-60

103. Eddington, A. S. 1915. MNRAS 76: 572-85

104. Efstathiou, G., Ellis, R. S., Carter, D. 1982. MNRAS 201: 975-90

105. Efstathiou, G., Silk, J. 1983. Fundament. Cosmic Phys. 9: 1-137

106. Evans, N. W. 1988. Separability and integrability in stellar dynamics. $\mathrm{PhD}$ thesis. Cambridge Univ., Cambridge

107. Evans, N. W. 1990. Intern. J. Computer. Math. 34: 105-12

108. Evans, N. W., de Zeeuw, P. T., LyndenBell, D. 1990. MNRAS 244: 111-29

109. Evans, N. W., Lynden-Bell, D. 1989. MNRAS 236: 801-16

110. Evans, N. W., Lynden-Bell, D. 1991. $M N R A S$. Submitted

111. Fabbiano, G. 1989. Annu. Rev. Astron. Astrophys. 27: 87-138

112. Faber, S. M., Dressler, A., Davies, S. L., Burstein, D., Lynden-Bell, D., et al. 1987. In Nearly Normal Galaxies, ed. S. M. Faber, pp. 175-83. Berlin: SpringerVerlag

113. Faber, S. M., Jackson, R. E. 1976. Ap. J. 204: 668-83

114. Faber, S. M., Wegner, G., Burstein, D., Davies, R. L., Dressler, A., et al. 1989. Ap. J. Suppl. 69: 763-808

115. Farouki, R. T., Shapiro, S. L. 1982. Ap. J. 259: 103-15

116. Fillmore, J. A. 1986. Astron. J. 91: 1096-1107

117. Fillmore, J. A., Boroson, T. A., Dressler, A. 1986. Ap. J. 302: 20833

118. Fillmore, J. A., Levison, H. F. 1989. Astron. J. 97: 57-68

119. Ford, H. C., Ciardullo, R., Jacoby, G. H., Hui, X. 1989. In IAU Symp. 131, 
Planetary Nebulae, ed. S. Torres-Peimbert, pp. 335-50. Dordrecht: Reidel

120. Forman, W., Jones, C., Tucker, W. 1985. Ap. J. 293: 102-19

121. Franx, M. 1988. MNRAS 231: 285 308

122. Franx, M., Illingworth, G. D. 1988. Ap. J. Lett. 327: L55-60

123. Franx, M., Illingworth, G. D. 1990. Ap. J. Lett. 359: L41-45

124. Franx, M., Illingworth, G. D., de Zeeuw, P. T. 1991. Ap. J. In press

125. Franx, M., Illingworth, G. D., Heckman, T. M. 1989. Ap. J. 344: 613-36

126. Franx, M., Illingworth, G. D., Heckman, T. M. 1989. Astron. J. 98: 538-76

127. Freeman, K. C. 1966. MNRAS 133 . 47-62

128. Freeman, K. C. 1966. MNRAS 134: 114

129. Freeman, K. C. 1966. MNRAS 134: 15-23

130. Freeman, K. C. 1975. In Stars and Stellar Systems, Vol. 9, Galaxies and the Universe, ed. A. Sandage, M. Sandage, J. Kristian, pp. 409-507. Chicago: Univ. Chicago Press

131. Freeman, K. C. 1990. In Dynamics and Interactions of Galaxies, ed. R. Wielen, pp. 36-47. Berlin: Springer-Verlag

132. Fridman, A. M., Polyachenko, V. L. 1984. Physics of Gravitating Systems. New York: Springer-Verlag

133. Galletta, G. 1980. Astron. Astrophys. 81: 179-81

134. Gerhard, O. E. 1981. MNRAS 197: 179-208

135. Gerhard, O. E. 1985. Astron. Astrophys. 151: 279-96

136. Gerhard, O. E. 1986. MNRAS 219: 373-86

137. Gerhard, O. E. 1987. In Ref. 83, pp. $241-48$

138. Gerhard, O. E. 1989. In Dynamics of Dense Stellar Systems, ed. D. R. Merritt, pp. 61-68. Cambridge: Cambridge Univ. Press

139. Gerhard, O. E. 1991. MNRAS. In press

140. Gerhard, O. E., Binney, J. J. 1985. MNRAS 216: 467-502

141. Gerhard, O. E., Vietri, M. 1986. MNRAS 223: 377-89

142. Gerhard, O. E., Vietri, M. 1987. In Ref. 83 , pp. 401-2

143. Gerhard, O. E., Vietri, M., Kent, S. M. 1989. Ap. J. Lett. 345: L33-36

144. Goodman, J. 1988. Ap. J. 329: 612-17

145. Goodman, J., Schwarzschild, M. 1981. Ap. J. 245: 1087-93

146. Gott, J. R. 1977. Annu. Rev. Astron. Astrophys. 15: 235-66

147. Deleted in proof

148. Gunn, J. E. 1986. In The Galaxy, ed.
G. Gilmore, R. Carswell, pp. 413-29. Dordrecht: Reidel

149. Gunn, J. E. 1988. In ASP conference series, Vol. 4, The Extragalactic Distance Scale, ed. S. van den Bergh, C. J. Pritchet, pp. 344-59. Provo: Brigham Young Univ. Print Serv.

150. Harris, W. E. 1988. In IAU Symp. 126, Globular Cluster Systems in Galaxies, ed. J. E. Grindlay, A. G. Davis-Philip, pp. 237-53. Dordrecht: Kluwer

151. Hasan, H., Norman, C. 1990. Ap. J. 361: 69-77

152. Heckman, T. M., Armus, L., Miley, G. K. 1991. Ap. J. In press

153. Heiligman, G. M., Schwarzschild, M. 1979. Ap.J. 233: 872-76

154. Heisler, J., Merritt, D., Schwarzschild, M. 1982. Ap. J. 258: 490-98

155. Held, E. V., Mould, J. R., de Zeeuw, P. T. 1990. Astron. J. 100: 415-19

156. Hernquist, L. 1989. Nature 340: 68791

157. Hernquist, L. 1990. In Dynamics and Interactions of Galaxies, ed. R. Wielen, pp. 108-17. Berlin: Springer-Verlag

158. Hesser, J. E., Harris, H. C., van den Bergh, S., Harris, G. L. M. 1984. Ap. J. 276: 491-508

159. Huchra, J. 1988. In IAU Symp. 126 , Globular Cluster Systems in Galaxies, ed. J. E. Grindlay, A. G. Davis-Philip, pp. 255-68. Dordrecht: Kluwer

160. Hunter, C. 1974. MNRAS 166: 633-48

161. Hunter, C. 1975. In IAU Symp. 69, Dynamics of Stellar Systems, ed. A. Hayli, pp. 195-203. Dordrecht: Reidel

162. Hunter, C. 1975. Astron. J. 80: 783-93

163. Hunter, C. 1977. Astron. J. 82: 271-82

164. Hunter, C. 1988. In Applied Mathematics, Fluid Mechanics, Astrophysics, ed. D. Benney, F. H. Shu, C. Yuan, pp. 350-57. Singapore: World Scientific

165. Hunter, C. 1988. In Integrability in Dynamical Systems, Ann. NY Acad. Sci. 536: 25-32

166. Hunter, C. 1990. In Galactic Models, Ann. NY Acad. Sci. 596: 187-97

167. Hunter, C., de Zeeuw, P. T. 1991. Ap. $J$. In press

168. Hunter, C., de Zeeuw, P. T., Park, Ch., Schwarzschild, M. 1990. Ap. J. 363: 367-90

169. Illingworth, G. D. 1977. Ap. J. 218: L43-47

170. Illingworth, G. D., Franx, M. 1989. In Dynamics of Dense Stellar Systems, ed. D. R. Merritt, pp. 13-23. Cambridge: Cambridge Univ. Press

171. Illingworth, G. D., Schechter, P. L. 1982. Ap. J. 256: 481-96

172. Innanen, K. A., Papp, K. A. 1977. Astron. J. 82: 322-28 
173. Jacobi, C. G. J. 1866. Vorlesungen über Dynamik. Berlin: Reimer

174. Jaffe, W. 1987. In Ref. 83, pp. 511-12

175. Jarvis, B. J. 1987. Astron. J. 94: 30-42

176. Jarvis, B. J., Dubath, P. 1988. Astron. Astrophys. Lett. 201: L33-36

177. Jarvis, B. J., Freeman, K. C. 1985. Ap. J. 295: 314-23

178. Jarvis, B. J., Freeman, K. C. 1985. Ap. J. 295: 324-39

179. Jeans, J. H. 1915. MNRAS 76: 71-84

180. Jeans, J. H. 1922. MNRAS 82: 122-32

181. Jedrzejewski, R. I., Schechter, P. L. 1988. Ap. J. Lett. 330: L87-91

182. Jedrzejewski, R. I., Schechter, P. L. 1989. Astron. J. 98: 147-65

183. Kang, H., Shapiro, P. R., Fall, S. M., Rees, M. J. 1990. Ap. J. 363: 488-98

184. Katz, N., Richstone, D. O. 1985. Ap. J. 296: 331-35

185. Kent, S. M. 1989. Astron. J. 97: 161421

186. Kent, S. M. 1990. In ASP conference series, Vol. 10, Evolution of the Universe of Galaxies, ed. R. G. Kron, pp. 10918. Provo: Brigham Young Univ. Print Serv.

187. Kim, D. W., Guhathakurta, P., van Gorkom, J. H., Jura, M., Knapp, G. R. 1988. Ap. J. 330: 684-94

188. Kondrat'ev, B. P., Ozernoy, L. M. 1979. Sov. Astron. Lett. 5: 37-39

189. Kormendy, J. 1982. In Morphology and Dynamics of Galaxies, ed. L. Martinet, M. Mayor, pp. 115-288. Sauverny: Geneva Observatory

190. Kormendy, J. 1984. Ap. J. 287: 577-85

191. Kormendy, J. 1988. Ap. J. 325: 128-41

192. Kormendy, J. 1988. Ap. J. 335: 40-56

193. Kormendy, J., Djorgovski, S. 1989. Annu. Rev. Astron. Astrophys. 27: 23577

194. Kormendy, J., Illingworth, G. D. 1982. Ap. J. 256: 460-80

195. Kormendy, J., Illingworth, G. D. 1983. Ap. J. 265: 632-42

196. Kormendy, J., Westphal, D. J. 1989. Ap. J. 338: 752-60

197. Kuzmin, G. G. 1956. Astr. Zh. 33: 2745

198. Kuzmin, G. G. 1973. In Dynamics of Galaxies and Clusters, ed. T. B. Omarov, pp. 71-75. Alma Ata: Akad. Nauk Kaz. SSR [Transl. in Ref. 83, pp. 553-57]

199. Kuzmin, G. G., Kutuzov, S. A. 1962. Byull. Abastumanskaya Astrofiz. Obs. 27: $82-88$

200. Lake, G. 1979. In Photometry, Kinematics and Dynamics of Galaxies, ed. D. S. Evans, pp. 381-86. Austin: Univ. Texas

201. Lake, G. 1981. Ap. J. 243: 111-20
202. Lake, G. 1981. Ap. J. 243: 121-26

203. Lake, G., Dressler, A. 1986. Ap. J. 310: 605-12

204. Lake, G., Schommer, R. A., van Gorkom, J. H. 1987. Ap. J. 314: 5769

205. Larson, R. B. 1974. MNRAS 166: 585 616

206. Larson, R. B. 1990. Publ. Astr. Soc. Pac. 102: 709-22

207. Lauer, T. R. 1985. Ap. J. 292: 104-21

208. Lees, J. F. 1991. In Warped Disks and Inclined Rings around Galaxies, ed. S. Casertano, P. Sackett, F. H. Briggs, pp. 50-59. Cambridge: Cambridge Univ. Press

209. Lees, J. F., Knapp, G. R., Rupen, M. P., Phillips, T. G. 1990. Ap. J. In press

210. Lees, J. F., Schwarzschild, M. 1991. Ap. $J$. In press

211. Levison, H. F. 1987. Ap. J. Lett. 320: L93-97

212. Levison, H. F., Duncan, M. J., Smith, B. F. 1990. Ap. J. 363: 66-78

213. Levison, H. F., Richstone, D. O. 1985. Ap. J. 295: $340-48$

214. Levison, H. F., Richstone, D. O. 1985. Ap. J. 295: 349-57

215. Levison, H. F., Richstone, D. O. 1987. Ap. J. 314: 476-92

216. Londrillo, P., Messina, A. 1990. MNRAS 242: 595-605

217. Londrillo, P., Messina, A., Stiavelli, M. 1991. $M N R A S$. In press

218. Lynden-Bell, D. 1960. Stellar and galactic dynamics. PhD thesis. Cambridge: Cambridge Univ.

219. Lynden-Bell, D. 1962. MNRAS 123: 447-58

220. Lynden-Bell, D. 1962. MNRAS 124: 19

221. Lynden-Bell, D. 1962. MNRAS 124: 95-123

222. Lynden-Bell, D. 1967. MNRAS 136: 101-21

223. Magnenat, P. 1982. Astron. Astrophys. 108: 89-94

224. Marchant, A. B., Olson, D. W. 1979. Ap. J. Lett. 230: L157-59

225. Martinet, L., de Zeeuw, P. T. 1988. Astron. Astrophys. 206: 269-78

226. Martinet, L., Mayer, F. 1975. Astron. Astrophys. 44: 45-57

227. Martinet, L., Pfenniger, D. 1987. Astron. Astrophys. 173: 81-85

228. May, A., Binney, J. J. 1986. MNRAS 221: $13 \mathrm{P}-18$

229. May, A., van Albada, T. S. 1984. MNRAS 209: 15-23

230. May, A., van Albada, T. S., Norman, C. A. 1985. MNRAS 214: 131-36

231. McElroy, D. B. 1983. Ap. J. 270: 485506 
232. McGlynn, T. A. 1984. Ap. J. 281: 1330

233. Merrifield, M. R., Kent, S. M. 1990. Astron. J. 99: 1548-57

234. Merritt, D. R. 1980. Ap. J. Suppl. 43: 435-56

235. Merritt, D. R. 1982. Astron. J. 87: 1279-87

236. Merritt, D. R. 1985. Astron. J. 90: 1023-37

237. Merritt, D. R. 1987. Ap. J. 313: $121-$ 35

238. Merritt, D. R. 1987. Ap. J. 319: 55-60

239. Merritt, D. R. 1987. In Ref. 83, pp. 315-29

240. Merritt, D. R. 1988. Astron. J. 95: 49699

241. Merritt, D. 1988. In ASP Conf. Ser., Vol. 5, The Minnesota Lectures on Clusters of Galaxies and Large-Scale Structure, ed. J. M. Dickey, pp. 175-96. Provo: Brigham Young Univ. Print Serv.

242. Merritt, D. R. 1990. In Galactic Models, Ann. NY Acad. Sci. 596: 15057

243. Merritt, D. R., Aguilar, L. A. 1985. MNRAS 217: 787-804

244. Merritt, D. R., de Zeeuw, P. T. 1983. Ap. J. Lett. 267: L19-L23

245. Merritt, D. R., Hernquist, L. 1991. Ap. $J$. In press

246. Merritt, D. R., Stiavelli, M. 1990. Ap. J. 358: 399-417

247. Merritt, D. R., Tremaine, S. D., Johnstone, D. 1989. MNRAS 236: 829-41

248. Miller, R. H. 1978. Ap. J. 223: 122-28

249. Miller, R. H. 1982. Ap. J. 254: 75-76

250. Miller, R. H., Smith, H. F. 1979. $A p$. J. 227: 407-14

251. Miller, R. H., Smith, H. F. 1979. $A p$. J. 227: 785-97

252. Miralda-Escudé, J., Schwarzschild, M. 1989. Ap. J. 339: 752-62

253. Monet, D. G., Richstone, D. O., Schechter, P. L. 1982. Ap. J. 245: 45458

254. Morse, P. M., Feshbach, H. 1953. Methods of Mathematical Physics. New York: McGraw-Hill

255. Mould, J. R., Han, M., Bothun, G. 1989. Ap. J. 347: 112-17

256. Mould, J. R., Oke, J. B., de Zeeuw, P. T., Nemec, J. M. 1990. Astron. J. 99: $1823-30$

257. Mulder, W. A. 1983. Astron. Astrophys. 121: 91-96

258. Mulder, W. A., Hooimeyer, J. R. A 1984. Astron. Astrophys. 134: 158-70

259. Nagai, R., Miyamoto, M. 1976. Publ. Astron. Soc. Jpn. 28: 1-17

260. Negroponte, J., White, S. D. M. 1983. MNRAS 205: 1009-29
261. Newton, A. J., Binney, J. J. 1984. MNRAS 210: 711-30

262. Nieto, J.-L., Bender, R., Davoust, E., Prugniel, P. 1990. Astron. Astrophys. 230: L17-20

263. Norman, C. A., May, A., van Albada, T. S. 1985. Ap. J. 296: 20-34

264. Ostriker, J. P. 1980. Comm. Astrophys. 8: $177-78$

265. Palmer, P. L., Papaloizou, J. 1987. MNRAS 224: 1043-54

266. Palmer, P. L., Papaloizou, J. 1988. MNRAS 231: 935-52

267. Palmer, P. L., Papaloizou, J. 1990. MNRAS 243: 263-66

268. Palmer, P. L., Papaloizou, J., Allen, A. J. 1989. MNRAS 238: 1281-1318

269. Palmer, P. L., Papaloizou, J., Allen, A. J. 1990. MNRAS 246: 415-32

270. Peletier, R. F., Davies, R. L., Illingworth, G. D., Davies, L. E., Cawson, M. 1990. Astron. J. 100: 1091-1142

271. Petrou, M. 1983. MNRAS 202: 1195 1208

272. Petrou, M. 1983. MNRAS 202: 120920

273. Pfenniger, D. 1984. Astron. Astrophys. 134: 373-86

274. Pfenniger, D. 1984. Astron. Astrophys. 141: 171-88

275. Pfenniger, D. 1986. Astron. Astrophys. 165: 74-83

276. Pfenniger, D., de Zeeuw, P. T. 1989. In Dynamics of Dense Stellar Systems, ed. D. Merritt, pp. 81-88. Cambridge: Cambridge Univ. Press

277. Pierce, M., Tully, R. B. 1988. Ap. J. 330: 579-95

278. Polyachenko, V. L. 1987. In Ref. 83, pp. 301-14

279. Polyachenko, V. L., Shukhman, I. G. 1981. Sov. Astron. 25: 533-41

280. Prendergast, K. H., Tomer, E. 1970. Astron. J. 75: 674-79

281. Prieur, J.-L. 1990. In Dynamics and Interactions of Galaxies, ed. R. Wielen, pp. 72-83. Berlin: Springer-Verlag

282. Quinn, P. J. 1984. Ap. J. 279: 596-609

283. Raimond, E., Faber, S. M., Gallagher, J. S., Knapp, G. R. 1981. Ap. J. 246: 708-21

284. Rees, M. J. 1984. Annu. Rev. Astron. Astrophys. 22: 471-506

285. Rees, M. J., Ostriker, J. 1977. MNRAS 179: $541-52$

286. Richstone, D. O. 1979. Ap. J. 234: 82528

287. Richstone, D. O. 1980. Ap. J. 238: 1039

288. Richstone, D. O. 1982. Ap. J. 252: 496507

289. Richstone, D. O. 1984. Ap. J. 281:100 11 
290. Richstone, D. O. 1987. In Ref. 83, pp. 261-69

291. Richstone, D. O., Bower, G., Dressler, A. 1990. Ap. J. 353: 118-22

292. Richstone, D. O., Tremaine, S. D. 1984. Ap. J. 286: 27-37

293. Richstone, D. O., Tremaine, S. D. 1986. Astron. J. 92: 72-74

294. Richstone, D. O., Tremaine, S. D. 1988. Ap. J. 327: 82-88

295. Rix, H., White, S. D. M. 1990. Ap. J. 362: 52-58

296. Rowley, G. 1988. Ap. J. 331: 124-34

297. Ryden, B. S., Gunn, J. E. 1987. Ap. J. 318: 15-31

298. Saaf, A. 1968. Ap. J. 154: 483-98

299. Saha, P. 1991. MNRAS 248: 494-502

300. Sarazin, C. L. 1987. In Ref. 83, pp. 17988

301. Sargent, W. L. W., Young, P. J., Boksenberg, A., Shortridge, K., Lynds, C. R., Hartwick, F. D. A. 1978. Ap. J. 221: 731-44

302. Satoh, C. 1980. Publ. Astron. Soc. Jpn. 32: 41-62

303. Schechter, P. L. 1987. In Ref. 83, pp. 217-28

304. Schechter, P. L., Gunn, J. 1979. Ap. J. 229: 472-84

305. Schwarzschild, M. 1979. Ap. J. 232: 236-47

306. Schwarzschild, M. 1982. Ap. J. 263: 599-610

307. Schwarzschild, M. 1987. In Chaotic Phenomena in Astrophysics, Ann. NY Acad. Sci. 497: 16-21

308. Schweizer, F. 1980. Ap. J. 237: 303-18

309. Schweizer, F. 1982. Ap. J. 252: 455-60

310. Schweizer, F. 1990. In Dynamics and Interactions of Galaxies, ed. R. Wielen, pp. 60-71. Berlin: Springer-Verlag

311. Schweizer, F., van Gorkom, J. H., Seitzer, P. 1989. Ap. J. 338: 770-88

312. Scorza, C., Bender, R. 1990. Astron. Astrophys. 235: 49-54

313. Silk, J. 1977. Ap. J. 211: 638-48

314. Smith, B. F., Miller, R. H. 1982. Ap. J. 257: 103-9

315. Sparke, L. S. 1986. MNRAS 219: 65770

316. Sparke, L. S., Sellwood, J. A. 1987. MNRAS 225: 653-75

317. Stäckel, P. 1890. Math. Ann. 35: 91103

318. Stäckel, P. 1891. Ueber die Integration der Hamilton-Jacobischen Differentialgleichungen Mittelst Separation der Variabeln. Halle: Habilitationschrift

319. Stanford, A., Balcells, M. 1990. Ap. J. 355: 59-69

320. Statler, T. S. 1987. Ap. J. 321: 113-52

321. Steiman-Cameron, T. Y., Durisen, R. H. 1982. Ap. J. Lett. 263: L51-54
322. Steiman-Cameron, T. Y., Durisen, R. H. 1988. Ap. J. 325: 26-48

323. Stiavelli, M., Bertin, G. 1985. MNRAS 217: 735-52

324. Stiavelli, M., Bertin, G. 1987. MNRAS 229: 61-71

325. Stiavelli, M., Sparke, L. S. 1991. Ap. J. In press

326. Teuben, P. J. 1987. MNRAS 227: 81541

327. Teuben, P. J., Sanders, R. H. 1985. MNRAS 212: 257-73

328. Tonry, J. L. 1981. Ap. J. Lett. 251: L15

329. Tonry, J. L. 1983. Ap. J. 266: 58-68

330. Tonry, J. L. 1987. Ap. J. 322: 632-42

331. Toomre, A. 1964. Ap. J. 139: 1217-38

332. Toomre, A. 1977. In The Evolution of Galaxies and Stellar Populations, ed. B. M. Tinsley, R. B. Larson, pp. 401-16. New Haven: Yale Univ. Observatory

333. Toomre, A. 1982. Ap. J. 259: 535-43

334. Toomre, A., Toomre, J. 1972. Ap. J. 178: 623-66

335. Tremaine, S. D. 1987. In Ref. 83, pp. $367-73$

336. Tremaine, S. D., Hénon, M., LyndenBell, D. 1986. MNRAS 219: 285-97

337. Trinchieri, G., Fabbiano, G., Canizares, C. R. 1986. Ap. J. 310: 637-59

338. Tully, R. B., Fisher, J. R. 1977. Astron. Astrophys. 54: 661-73

339. Udry, S., Pfenniger, D. 1988. Astron. Astrophys. 198: 135-49

340. van Albada, T. S. 1982. MNRAS 201: 939-55

341. van Albada, T. S. 1987. In Ref. 83, pp. 291-99

342. van Albada, T. S., Kotanyi, C. G., Schwarzschild, M. 1982. MNRAS 198: 303-10

343. van den Bergh, S. 1990. In Dynamics and Interactions of Galaxies, ed. R. Wielen, pp. 492-98. Berlin: SpringerVerlag

344. van der Marel, R. P., Binney, J. J., Davies, R. L. 1990. MNRAS 245: 58296

345. Vandervoort, P. O. 1979. Ap. J. 232: 91-105

346. Vandervoort, P. O. 1980. Ap. J. 240: 478-87

347. Vandervoort, P. O. 1980. Ap. J. 241: 316-33

348. Vandervoort, P. O. 1984. Ap. J. 287: $475-86$

349. Vandervoort, P. O. 1989. Ap. J. 341: 105-12

350. Vandervoort, P. O., Welty, D. E. 1982. Ap. J. 263: 654-71

351. van Gorkom, J. H. 1991. In Morphological and Physical Classification of Galaxies, ed. G. Busarello, M. 
Capaccioli, G. Longo. Dordrecht: Kluwer. In press

352. van Gorkom, J. H., Knapp, G. R., Raimond, E., Faber, S. M., Gallagher, J. S. 1986. Astron. J. 91: 791-807

353. van Gorkom, J. H., van der Hulst, J. M., Haschick, A. D., Tubbs, A. D. 1990. Astron. J. 99: 1781-88

354. Vietri, M. 1986. Ap. J. 306: 48-63

355. Vietri, M. 1990. Personal communication

356. Vietri, M., Schwarzschild, M. 1983. Ap. J. 269: 487-99

357. Villumsen, J. V. 1982. MNRAS 199: 493-516

358. Villumsen, J. V. 1984. Ap. J. 284: 7589

359. Wagner, S. J. 1990. In Dynamics and Interactions of Galaxies, ed. R. Wielen, pp. 244 48. Berlin: Springer-Verlag

360. Wagner, S. J., Bender, R., Möllenhof, C. 1988. Astron. Astrophys. Lett. 195: L5-8

361. Weinberg, M. D. 1989. MNRAS 239: 549-70

362. Weinberg, M. D. 1991. Ap.J. 368: 6678

363. White, S. D. M. 1979. MNRAS 189: $831-52$

364. White, S. D. M. 1987. In Ref. 83, pp. 339-51

365. White, S. D. M. 1989. In The Epoch of
Galaxy Formation, ed. C. S. Frenk et al., pp. 15-29. Dordrecht: Kluwer

366. White, S. D. M., Davis, M., Efstathiou, G., Frenk, C. S. 1987. Nature 330: 45153

367. White, S. D. M., Narayan, R. 1987. MNRAS 229: 103-17

368. White, S. D. M., Rees, M. J. 1978. MNRAS 183: 341-58

369. Whitmore, B. C., Bell, M. 1988. Ap. J. 324: $741-48$

370. Whitmore, B. C., Rubin, V. C., Ford, W. K. 1984. Ap. J. 287: 66-79

371. Wilkinson, A., James, R. A. 1982. MNRAS 199: 171-96

372. Wilkinson, A., Sharples, R. M., Fosbury, R. A. E., Wallace, P. T. 1986. MNRAS 218: 297-329

373. Williams, T. B. 1981. Ap. J. 244: 45866

374. Williams, T. B., Schwarzschild, M. 1978. Ap. J. 227: 56-63

375. Wilson, C. P. 1975. Astron. J. 80: 17587

376. Wyse, R. F., Jones, B. J. T. 1984. Ap. J. 286: 88-96

377. Young, P. J., Westphal, J. A., Kristian, J., Wilson, C. D., Landauer, F. P. 1978. Ap. J. 221: 721-30

378. Zurek, W. H., Quinn, P. J., Salmon, J. K. 1988. Ap. J. 330: 519-34 